\title{
BOUNDING THE RANK OF HERMITIAN FORMS AND RIGIDITY FOR CR MAPPINGS OF HYPERQUADRICS
}

\author{
DUSTY GRUNDMEIER, JIŘÍ LEBL, AND LIZ VIVAS \\ Dedicated to the memory of Professor M. Salah Baouendi
}

\begin{abstract}
Using Green's hyperplane restriction theorem, we prove that the rank of a Hermitian form on the space of holomorphic polynomials is bounded by a constant depending only on the maximum rank of the form restricted to affine manifolds. As an application we prove a rigidity theorem for $\mathrm{CR}$ mappings between hyperquadrics in the spirit of the results of Baouendi-Huang and Baouendi-Ebenfelt-Huang. Given a real-analytic CR mapping of a hyperquadric (not equivalent to a sphere) to another hyperquadric $Q(A, B)$, either the image of the mapping is contained in a complex affine subspace, or $A$ is bounded by a constant depending only on $B$. Finally, we prove a stability result about existence of nontrivial CR mappings of hyperquadrics. That is, as long as both $A$ and $B$ are sufficiently large and comparable, then there exist CR mappings whose image is not contained in a hyperplane. The rigidity result also extends when mapping to hyperquadrics in infinite dimensional Hilbert-space.
\end{abstract}

\section{INTRODUCTION}

A real-valued polynomial, or a real-analytic function, $r(z, \bar{z})$ on $\mathbb{C}^{n}$ can be regarded as a Hermitian form by considering the matrix of coefficients of the series. That is, in multi-index notation, write

$$
r(z, \bar{z})=\sum_{\alpha \beta} c_{\alpha \beta} z^{\alpha} \bar{z}^{\beta} .
$$

The matrix $C=\left[c_{\alpha \beta}\right]_{\alpha \beta}$ is Hermitian symmetric if and only if $r$ is real-valued ( $C$ is uniquely determined by $r$ ).

When we apply linear algebra terminology (such as rank, eigenvalues, signature, or positive semidefinite) to $r$, we simply refer to the underlying matrix $C$. When $r$ is a polynomial, the terminology is obvious. When $r$ is real-analytic, then $C$ is an infinite matrix. After possibly rescaling so that the series converges in a neighborhood of the closed unit polydisc, $C$ defines a Hermitian trace-class operator, and the terminology easily extends to the realanalytic case; see section 5. While the matrix depends on the point where we expand the series, we show that the rank does not change under a biholomorphic change of coordinates. In particular the rank does not depend on the point where we expand the series.

In order to state the first result, we define the rank of the restriction to an affine manifold. The Grassmannian $G(m, n)$ is the manifold of all $m$-dimensional linear subspaces of $\mathbb{C}^{n}$, and the affine Grassmannian $G_{m, n}$ is the manifold of all affine $m$-dimensional subspaces in $\mathbb{C}^{n}$. Notice that $G_{m, n}$ is an open set in $G(m+1, n+1)$.

Date: October 9, 2013.

The first author acknowledges support from NSF grants DMS 0838434 and NSF RTG-0602191.

The second author was in part supported by NSF grant DMS 0900885. 
Let $U \subset \mathbb{C}^{n}$ be a convex neighborhood of the origin, and define $U^{*}:=\{z: \bar{z} \in U\}$. Suppose that the series (1) converges in $U \times U^{*}$. Let $L$ be an affine $m$-plane in $\mathbb{C}^{n}$ (i.e. an element of $\left.G_{m, n}\right)$. Let $E: \mathbb{C}^{m} \rightarrow \mathbb{C}^{n}$ be an affine embedding of $L$ in $\mathbb{C}^{n}$. If $L$ intersects $U$, then define

$$
\left.\operatorname{rank} r\right|_{L}:=\operatorname{rank} r \circ E .
$$

It is not hard to show that the rank does not depend on the particular embedding $E$, and therefore the notation is well-defined. Furthermore as $U$ is convex, $U \cap L$ is always connected. If $L$ does not intersect $U$, then define

$$
\left.\operatorname{rank} r\right|_{L}:=-\infty
$$

Let $\mathbb{N}_{0}=\mathbb{N} \cup\{0\}$.

Theorem 1.1. Let $n \geq 2$ and let $1 \leq m \leq n-1$. Let $r(z, \bar{z})$ be a real-analytic function defined in a convex neighborhood $U \subset \mathbb{C}^{n}$ of the origin such that the complexified power series converges in $U \times U^{*}$. If

$$
\left.\sup _{L \in G_{m, n}} \operatorname{rank} r\right|_{L}<\infty
$$

then $\operatorname{rank} r<\infty$.

Moreover, there exists a function $R_{m, n}: \mathbb{N}_{0} \rightarrow \mathbb{N}_{0}$ such that for any such real-analytic $r$,

$$
\operatorname{rank} r \leq R_{m, n}\left(\left.\max _{L \in G_{m, n}} \operatorname{rank} r\right|_{L}\right) .
$$

It is worthwhile to state the theorem for bihomogeneous polynomials. A polynomial $r(z, \bar{z})$ is said to be bihomogeneous of bidegree $(d, d)$ if $r(t z, \bar{z})=r(z, t \bar{z})=t^{d} r(z, \bar{z})$. When $r$ is a polynomial then the coefficient matrix is simply a finite matrix. The function $R_{m, n}$ in the following theorem is the same as above.

Theorem 1.2. Let $n \geq 2, z \in \mathbb{C}^{n+1}$, and $1 \leq m \leq n-1$. Then there exists a function $R_{m, n}: \mathbb{N}_{0} \rightarrow \mathbb{N}_{0}$ such that for any bihomogeneous polynomial $r(z, \bar{z})$,

$$
\operatorname{rank} r \leq R_{m, n}\left(\left.\max _{L \in G(m+1, n+1)} \operatorname{rank} r\right|_{L}\right) .
$$

As an application of Theorem 1.1, we prove a rigidity result for mappings of hyperquadrics. The hyperquadric $Q(a, b) \subset \mathbb{C}^{a+b}$ is the set defined by

$$
Q(a, b):=\left\{z \in \mathbb{C}^{a+b}: \sum_{j=1}^{a}\left|z_{j}\right|^{2}-\sum_{j=a+1}^{a+b}\left|z_{j}\right|^{2}=1\right\} .
$$

When $b=0$, then $Q(a, 0)$ is simply the sphere. Hyperquadrics and spheres are the CR analogues of flat euclidean space from Riemannian geometry. In CR geometry, however, there is no analogue of the Nash embedding theorem. Therefore, a natural question in CR geometry is to study the $\mathrm{CR}$ mappings $f: U \rightarrow Q(A, B)$ for a $\mathrm{CR}$ manifold $U$. In this paper we take an open subset $U \subset Q(a, b)$. We study hyperquadrics $Q(a, b)$ that are not equivalent to the sphere. By a theorem of Lewy [17] a CR function on $Q(a, b)$ extends to a holomorphic function of both sides of $Q(a, b)$. It is therefore enough to consider real-analytic $\mathrm{CR}$ mappings, or in other words restrictions of holomorphic mappings. Therefore we study holomorphic mappings of a neighborhood of $U$ in $\mathbb{C}^{a+b}$ to $\mathbb{C}^{A+B}$ that take $U \subset Q(a, b)$ to $Q(A, B)$. See the books [2,5] for more background information. 
When considering mappings between hyperquadrics, we need to consider problems of positivity rather than just rank. See [8,9] for more on positivity conditions.

After a linear fractional change of coordinates we can assume that $a>b$ and $A>B$. It is possible that the change of coordinates has a pole on $U$, but as $Q(a, b)$ is a real hypersurface, there still has to exist a perhaps smaller dense open set $V \subset U$ such that $f: V \rightarrow Q(A, B)$ is a CR mapping.

The study of CR mappings between spheres has a long history. When the target is also a sphere $(B=0)$ and the codimension $A-a$ is small, then certain strong rigidity results can be proven; see for example [14] and the references within. Forstnerič proved that sufficiently smooth CR mappings of spheres must be rational of degree bounded by a constant depending only on the dimensions involved [10].

When the target dimension is large, there is less rigidity. When both the source and target are spheres, increasing the dimension of the target always adds new CR mappings as long as the dimension is large enough; see [6]. Furthermore, if the source is a sphere and the target is a hyperquadric not equivalent to a sphere, then for large target dimension not only do we always get new mappings, but we get new rational mappings of arbitrarily large degree; see 77.

We therefore concentrate on the case when $b, B \geq 1$. Baouendi and Huang [4] proved that if $b=B$, then $f$ must be a linear embedding. Later, Baouendi, Ebenfelt, and Huang [3] proved that if $B<2 b$, then after a change of coordinates $f$ can be written as

$$
\left(z_{1}, \ldots, z_{n}\right) \mapsto\left(z_{1}, \ldots, z_{a}, \psi(z), 0, z_{a+1}, \ldots, z_{a+b}, \psi(z), 0\right),
$$

where an arbitrary CR mapping $\psi$ and 0 are vector-valued functions with the right number of components. In particular, unless $a=A$ and $b=B, f(U)$ is contained in a complex hyperplane, where by a complex hyperplane we mean a complex affine manifold of complex codimension one. The mapping (8) can be written, after an affine change of coordinates, as the identity mapping direct sum an arbitrary CR mapping going into a lower dimensional ambient space.

For any mapping $f$ of hyperquadrics, if $f(U)$ lies in a complex hyperplane, then we apply an affine change of coordinates and obtain a mapping $\tilde{f}$ from $U$ to $Q\left(A^{\prime}, B^{\prime}\right) \times \mathbb{C}^{k}$ for some $A^{\prime} \leq A$ and $B^{\prime} \leq B$. We can study the first $A^{\prime}+B^{\prime}$ components of $\tilde{f}$ as a mapping from $Q(a, b)$ to $Q\left(A^{\prime}, B^{\prime}\right)$, as the last $k$ components of $\tilde{f}$ are arbitrary. Therefore, it is natural to study those mappings where $f(U)$ is not contained in a complex hyperplane.

Theorem 1.3. Let $a>b \geq 1, U \subset Q(a, b)$ be a connected open set, and $f: U \rightarrow Q(A, B)$ be a real-analytic $C R$ mapping such that $f(U)$ does not lie in a complex hyperplane, then

$$
A \leq N(a, b, B)
$$

where $N=N(a, b, B)$ is a constant depending only on $a, b$, and $B$.

Note that the hypotheses on $Q(a, b)$ mean that $Q(a, b)$ is not equivalent to a sphere. If $Q(a, b)$ were equivalent to a sphere, there is no rigidity. In this sense, the theorem is optimal.

We are interested in what happens when $B \geq 2 b$. In this situation, there do exist nontrivial mappings that do not map to a hyperplane. See sections 8 and 9 for a general method of constructing mappings.

The bound that we obtain on $A$ is not sharp. For example when $a=2$ and $b=1$, it is possible to use the method in this paper to obtain an explicit bound

$$
A \leq K_{1,3}(B+1)=K_{3}\left(K_{2}(B+1)\right) .
$$


See section 3 for the definition of $K_{n}(k)$. When $B=1$ we obtain $A \leq 4$, though we know that $A=2$ by the result of Baouendi-Huang. We dispense with finding explicit formulas for $N(a, b, B)$; however, we briefly describe the asymptotics. In section 5 we show that $K_{n}(k) \leq \widetilde{K}_{n}(k)=\frac{n-1}{n} k^{\frac{n}{n-1}}+\frac{1}{n} k$, and furthermore, in section 6, we show

$$
\begin{aligned}
A \leq N(a, b, B) & \leq K_{a+b} \circ K_{a+b-1} \circ \cdots \circ K_{b+1}(B+1) \\
& \leq \widetilde{K}_{a+b} \circ \widetilde{K}_{a+b-1} \circ \cdots \circ \widetilde{K}_{b+1}(B+1) \\
& \left.=\left(\prod_{\ell=0}^{a-1}\left(\frac{a+b-1-\ell}{a+b-\ell}\right)^{\frac{a+b}{a+b-\ell}}\right) B^{\frac{a+b}{b}}+\text { (lower order terms in } B\right) .
\end{aligned}
$$

Thus the bound $N(a, b, B)$ is itself bounded by a polynomial in $B$ of degree at most $\frac{a+b}{b}$.

Unlike in the sphere to sphere case, there do exist non-rational mappings, and rational mappings of arbitrarily high degree. In section 9, we prove a stability result about existence of hyperquadric mappings that do not map into hyperplanes as long as both $A$ and $B$ are sufficiently large and comparable. The proof is constructive and all constructed mappings are monomial; that is, all components are single monomials.

The stability result discussed above shows that Theorem 1.3 is optimal in the sense that super-rigidity as studied by Baouendi-Huang and Baouendi-Ebenfelt-Huang only appears in small codimension. In general the best constant $N$ must grow asymptotically at least as a linear function of $B$. The methods explored in this paper do not readily give the best bound for $N$ as we have mentioned above, but we do obtain that $N$ asymptotically grows no faster than a polynomial in $B$.

Finally, we also note that the rigidity result extends when the target is an infinite dimensional hyperquadric $Q(\infty, B)$, where $B \in \mathbb{N}_{0} \cup\{\infty\}$; see section 7. Lempert [16] shows that any strictly pseudoconvex real-analytic compact hypersurface can be mapped into a sphere in a possibly infinite dimensional Hilbert space. We show that if $Q(a, b)$ is not equivalent to a sphere, then for a CR mapping $Q(a, b) \rightarrow Q(\infty, B)$ where the image is not contained in a hyperplane we must have $B=\infty$. However, we also construct mappings whose image is not contained in a hyperplane from a real hypersurface $M \subset \mathbb{C}^{n}$ with an indefinite nondegenerate Levi-form to $Q(\infty, B)$ for finite $B$.

The authors would like to acknowledge John D'Angelo for many useful conversations on the subject and suggestions related to this project. The second author would also like to thank Peter Ebenfelt for useful discussions on this subject. The authors are greatly indebted to the referee who pointed out several errors in the presentation of the results and helped uncover a gap in the proof of Lemma 5.6, which has been fixed. Finally the authors would like to acknowledge MSRI and AIM for holding workshops on the subject of CR complexity, which the authors attended and which led to the present project.

\section{THE BASIC SETUP}

We begin by recalling the relevant definitions and proving some basic results about restrictions of real-valued polynomials. Let $\langle\cdot, \cdot\rangle$ denote the standard Hermitian inner product. Let $A$ be a Hermitian symmetric matrix, then we call $\langle A z, z\rangle$ a Hermitian form where $z \in \mathbb{C}^{n+1}$.

Let

$$
\mathcal{Z}_{n, d}: \mathbb{C}^{n+1} \rightarrow \mathbb{C}^{\left(\begin{array}{c}
n+d \\
d
\end{array}\right)}
$$


be the mapping whose components are all the degree $d$ monomials in $n+1$ variables $z_{0}, z_{1}, \ldots, z_{n}$. That is, $\mathcal{Z}_{n, d}$ is the mapping given by

$$
z \mapsto\left(z_{0}^{d}, z_{0}^{d-1} z_{1}, \ldots, z_{n}^{d}\right)
$$

The mapping $\mathcal{Z}_{n, d}$ is called the Veronese mapping in $n+1$ variables of degree $d$.

Definition 2.1. Let $L$ be an $(m+1)$-dimensional subspace of $\mathbb{C}^{n+1}$. Let $E_{L}$ be a linear embedding of $L$ into $\mathbb{C}^{n+1}$. Define $S_{E_{L}}^{d}$ to be the linear mapping that makes the following diagram commute:

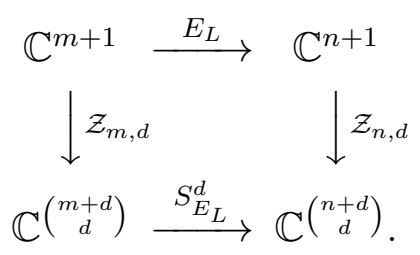

Define $T_{E_{L}}^{d}$ to be the matrix associated to the linear mapping $S_{E_{L}}^{d}$, then

$$
\mathcal{Z}_{n, d} \circ E_{L}=T_{E_{L}}^{d} \mathcal{Z}_{m, d}
$$

We often abbreviate $T_{L}^{d}$ for $T_{E_{L}}^{d}$ when the particular choice of embedding is not crucial.

Note that $T_{E_{L}}^{d}$ is not unique; it depends on the particular embedding $E_{L}$. However, if we have $E_{L}$ and $F_{L}$ two embeddings of $L$ then we have $T_{E_{L}}^{d}=\psi \circ T_{F_{L}}^{d}$, where $\psi$ is an isomorphism.

Lemma 2.2. Let $r(z, \bar{z})$ be a bihomogeneous polynomial defined for $z \in \mathbb{C}^{n+1}$. If $\phi$ is a linear change of coordinates of $\mathbb{C}^{n+1}$, then

$$
\operatorname{rank} r \circ \phi=\operatorname{rank} r \text {. }
$$

Proof. We prove a more general fact in Lemma 5.2.

The lemma shows that rank of $\left.r\right|_{E_{L}}$ does not depend on the choice of embedding $E_{L}$. We record this observation in the following lemma.

Lemma 2.3. Let $r(z, \bar{z})=\left\langle C \mathcal{Z}_{n, d}, \mathcal{Z}_{n, d}\right\rangle$, where $C$ is a Hermitian matrix, and suppose $n \geq 1$. Let $L$ be an $(m+1)$-dimensional subspace of $\mathbb{C}^{n+1}$. Let $E_{L}$ and $F_{L}$ be two embeddings of $L$ into $\mathbb{C}^{n+1}$. Then

$$
\operatorname{rank} T_{E_{L}}^{*} C T_{E_{L}}=\operatorname{rank} T_{F_{L}}^{*} C T_{F_{L}}
$$

Definition 2.4. Let $H_{\vec{c}}$ denote the hyperplane in $\mathbb{C}^{n+1}$ given by the following defining equation:

$$
z_{0}=c_{1} z_{1}+\cdots+c_{n} z_{n},
$$

where $\vec{c}=\left(c_{1}, c_{2}, \ldots, c_{n}\right)$. We embed $H_{\vec{c}}$ using $E_{H_{\vec{c}}}\left(z_{1}, \ldots, z_{n}\right)=\left(\sum_{j=1}^{n} c_{j} z_{j}, z_{1}, \ldots, z_{n},\right)$.

We abbreviate the notation for the restriction matrix $T_{H_{\vec{c}}}^{d}$ by $T_{\vec{c}}^{d}$.

The restriction matrix $T_{\vec{c}}^{d}$ is of size $\left(\begin{array}{c}n+d \\ d\end{array}\right) \times\left(\begin{array}{c}n+d-1 \\ d\end{array}\right)$.

To study the maximum rank of restrictions of bihomogeneous polynomials to hyperplanes, it suffices to study the hyperplanes of the form $H_{\vec{c}}$.

Lemma 2.5. Let $r(z, \bar{z})$ be a bihomogeneous polynomial defined for $z \in \mathbb{C}^{n+1}$ and suppose $n \geq 1$. Then

$$
\left.\max _{H} \operatorname{rank} r\right|_{H}=\left.\max _{\vec{c} \in \mathbb{C}^{n}} \operatorname{rank} r\right|_{H_{\vec{c}}}
$$

where $H$ ranges over all complex hyperplanes through the origin. 
Proof. The set of hyperplanes of the form $H_{\vec{c}}$ is dense in the Grassmannian $G(n, n+1)$. The rank of the restriction being bounded by a fixed integer is a holomorphic condition. Therefore $\left.\operatorname{rank} r\right|_{H}$ achieves the maximum on an open dense set in $G(n, n+1)$, and the conclusion follows.

\section{Green's Restriction Theorem}

The main technical tool in this paper is Green's hyperplane restriction theorem from [13]. We begin this section by recalling Macaulay representations and their basic properties. Then we give a precise statement of Green's theorem and translate the result into the language of this paper. In Lemma 5.6 we will prove a more general analytic version of Green's result using the ideas from the proof of the restriction theorem in 12 .

Given a non-negative integer $c$ and a positive integer $d$, then $c$ is uniquely expressed in the following form:

$$
c=\left(\begin{array}{c}
k_{d} \\
d
\end{array}\right)+\left(\begin{array}{c}
k_{d-1} \\
d-1
\end{array}\right)+\cdots+\left(\begin{array}{c}
k_{1} \\
1
\end{array}\right),
$$

where $0 \leq k_{1}<k_{2}<\cdots<k_{d}$. This expression is known as the $d$-th Macaulay representation of $c$. Following [13], we introduce the following notation:

$$
c_{<d>}=\left(\begin{array}{c}
k_{d}-1 \\
d
\end{array}\right)+\left(\begin{array}{c}
k_{d-1}-1 \\
d-1
\end{array}\right)+\cdots+\left(\begin{array}{c}
k_{1}-1 \\
1
\end{array}\right)
$$

where we define $\left(\begin{array}{l}a \\ b\end{array}\right)=0$ for $a<b$. Notice that $\cdot<d>$ is monotone increasing.

Theorem 3.1 (Green [13]). Let $E$ be a linear system in $H^{0}\left(\mathbb{C P}^{n}, \mathcal{O}(d)\right)$ and $E_{H}$ be the restriction of $E$ to a general hyperplane $H \in G(n, n+1)$. If $c$ is the codimension of $E$ in $H^{0}\left(\mathbb{C P}^{n}, \mathcal{O}(d)\right)$ and $c_{H}$ is the codimension of $E_{H}$ in $H^{0}\left(\mathbb{C P}^{n-1}, \mathcal{O}(d)\right)$, then

$$
c_{H} \leq c_{<d>}
$$

We seek a bound of the dimension of $E$ in terms of the generic dimension of the restriction. First we restate the theorem in terms of matrices. If $E$ is a linear system in $H^{0}\left(\mathbb{C P}^{n}, \mathcal{O}(d)\right)$ then we write: $E=\operatorname{span}\left\{s_{1}, s_{2}, \ldots, s_{m}\right\}$ where $s_{i} \in H^{0}\left(\mathbb{C P}^{n}, \mathcal{O}(d)\right)$. Since each $s_{i}$ is a degree $d$ homogeneous polynomial in $z_{0}, z_{1}, \ldots, z_{n}$, then we write $s_{i}=A_{i} \mathcal{Z}_{n, d}$, where each $A_{i}$ is a vector of the right dimension. Then we have $\operatorname{rank} E=\operatorname{rank} A$, where $A$ is the matrix whose rows are $A_{i}$.

If we restrict $E$ to a hyperplane $H$ we obtain

$$
\left.E\right|_{H}=\operatorname{span}\left\{\left.s_{1}\right|_{H},\left.s_{2}\right|_{H}, \ldots,\left.s_{m}\right|_{H}\right\} .
$$

Then $\left.s_{i}\right|_{H_{\vec{c}}}=A_{i} T_{\vec{c}}^{d} \mathcal{Z}_{n-1, d}$, and therefore

$$
\left.\operatorname{rank} E\right|_{H_{\vec{c}}}=\operatorname{rank} A T_{\vec{c}}^{d} .
$$

If we refer to a generic choice of $H$, then equivalently we refer to a generic set of $c_{i}$ 's, where we have: $\left.\operatorname{rank}_{\mathbb{C}} E\right|_{H_{\vec{c}}}=\operatorname{rank}_{\mathbb{C}\left[c_{1}, \ldots, c_{n}\right]} A T_{\vec{c}}^{d}$. That is, the rank of $\left.E\right|_{H_{\vec{c}}}$ over $\mathbb{C}$ is equal to the rank of $A T_{\vec{c}}^{d}$ over the polynomial ring $\mathbb{C}\left[c_{1}, \ldots, c_{n}\right]$.

Green's theorem can now be restated as follows: Let $A$ be any matrix of size $m \times\left(\begin{array}{c}n+d \\ d\end{array}\right)$, and $N=\operatorname{rank}_{\mathbb{C}} A$. Recall the matrix $T_{\vec{c}}^{d}$ is of size $\left(\begin{array}{c}n+d \\ d\end{array}\right) \times\left(\begin{array}{c}n-1+d \\ d\end{array}\right)$. Let $k=\operatorname{rank}_{\mathbb{C}\left[c_{1}, \ldots, c_{n}\right]} A T_{\vec{c}}^{d}$. Then we have the following relationship:

$$
G(n, d, N) \leq k,
$$


where

$$
G(n, d, N)=\left(\begin{array}{c}
n+d-1 \\
d
\end{array}\right)-\left(\left(\begin{array}{c}
n+d \\
d
\end{array}\right)-N\right)_{<d>} .
$$

In the following lemma, we show that the lower bound $G$ is constant in degree.

Lemma 3.2. If $N \leq\left(\begin{array}{c}n+d \\ d\end{array}\right), d \geq 1$, and $n \geq 2$, then $G(n, d, N)=G(n, d+1, N)$.

Proof. The lemma follows immediately from

$$
\left[\left(\begin{array}{c}
n+d+1 \\
d+1
\end{array}\right)-N\right]_{<d+1>}=\left(\begin{array}{c}
n+d-1 \\
d+1
\end{array}\right)+\left[\left(\begin{array}{c}
n+d \\
d
\end{array}\right)-N\right]_{<d>}
$$

By the lemma, we know that $G$ is constant in degree when it is defined. We also have $G$ is monotone in $N$ and $G\left(n, d,\left(\begin{array}{c}n+d \\ d\end{array}\right)\right)=\left(\begin{array}{c}n+d-1 \\ d\end{array}\right)$, hence the following bound is finite and well-defined for fixed $k$.

Definition 3.3. Fix $n$ and $k$. Define

$$
K_{n}(k):=\max \left\{N_{0} \in \mathbb{N}_{0}: G\left(n, d, N_{0}\right) \leq k, \text { where } d \text { is such that } N_{0} \leq\left(\begin{array}{c}
n+d \\
d
\end{array}\right)\right\} .
$$

Remark 3.4. The function $K_{n}(k)$ gives the sharp dimension bound from Green's theorem. The bound is achieved by restricting all the monomials in $n$ variables of degree $d$. In two dimensions, $K_{2}(k)$ has a particularly simple form:

$$
K_{2}(k)=\frac{k(k+1)}{2}
$$

In higher dimensions exact formulas are difficult, but we find a simple upper bound in Remark 5.7.

$$
K_{n}(k) \leq \frac{n-1}{n} k^{\frac{n}{n-1}}+\frac{1}{n} k .
$$

Note that equality holds when $n=2$.

The version of Green's theorem that we use in this paper is recorded in the following lemma. The important point is that the bound $K_{n}(k)$ does not depend on the degree $d$.

Lemma 3.5. Let $n \geq 2$. If $A \mathcal{Z}_{n, d}$ is a linear system in $H^{0}\left(\mathbb{C} \mathbb{P}^{n}, \mathcal{O}(d)\right)$ with rank $N$ and $k$ is the rank of the restriction to a general hyperplane; namely, $k=\operatorname{rank}_{\mathbb{C}\left[c_{1}, \ldots, c_{n}\right]} A T_{\vec{c}}^{d}$, then

$$
N \leq K_{n}(k) .
$$

The lemma gives an upper bound for $N$ that is independent of $d$ for fixed $k$ and $n \geq 2$. No such upper bound is possible if $n=1$.

\section{Restrictions of HeRmitian forms}

In this section, we prove Theorem 1.2. To illustrate the main idea of the proof we prove a weaker version of the main result for hyperplanes.

Lemma 4.1. Let $n \geq 2$ and $z \in \mathbb{C}^{n+1}$. There exists a function $R_{n}: \mathbb{N}_{0} \rightarrow \mathbb{N}_{0}$, such that if $r(z, \bar{z})$ is a real-valued bihomogeneous, then

$$
\operatorname{rank} r \leq R_{n}\left(\left.\max _{H} \operatorname{rank} r\right|_{H}\right),
$$

where $H$ ranges over all complex hyperplanes through the origin. 
Proof. Suppose $r$ is a nonzero bihomogeneous polynomial of bidegree $(d, d)$. We write $r(z, \bar{z})=\left\langle C \mathcal{Z}_{n, d}, \mathcal{Z}_{n, d}\right\rangle$, where $C$ is the coefficient matrix. Restricting $r$ to a hyperplane $H$, we have

$$
\left.r\right|_{H}(z, \bar{z})=\left\langle T_{H}^{*} C T_{H} \mathcal{Z}_{n-1, d}, \mathcal{Z}_{n-1, d}\right\rangle
$$

Let $k=\left.\max _{H} \operatorname{rank} r\right|_{H}=\max _{H} \operatorname{rank} T_{H}^{*} C T_{H}$ where $H$ ranges over all complex hyperplanes, then by Lemma 2.5 ,

$$
k=\left.\max _{\vec{c} \in \mathbb{C}^{n}} \operatorname{rank} r\right|_{H_{\vec{c}}}=\max _{\vec{c}} \operatorname{rank}\left(T_{\vec{c}}^{d}\right)^{*} C T_{\vec{c}}^{d}=\operatorname{rank}_{\mathbb{C}\left[\bar{c}_{1}, \ldots, \bar{c}_{n}, c_{1}, \ldots, c_{n}\right]}\left(T_{\vec{c}}^{d}\right)^{*} C T_{\vec{c}}^{d} .
$$

The strategy is to polarize (treat $c_{j}$ and $\bar{c}_{j}$ as separate variables) and use Lemma 3.5 twice.

Let $D=C T_{\vec{c}}^{d}, m=\operatorname{rank}_{\mathbb{C}} C$, and let $k_{1}=\operatorname{rank}_{\mathbb{C}\left[c_{1}, \ldots, c_{n}\right]} D$. By Lemma 3.5, we have

$$
m \leq K_{n}\left(k_{1}\right) .
$$

For a generic choice of $c_{1}, \ldots, c_{n}$, we have

$$
k_{1}=\operatorname{rank}_{\mathbb{C}} D=\operatorname{rank}_{\mathbb{C}} D^{*} .
$$

Likewise, polarizing and taking generic choices of $\bar{c}_{1}, \ldots, \bar{c}_{n}$, we have

$$
\operatorname{rank}_{\mathbb{C}\left[\bar{c}_{1}, \ldots, \bar{c}_{n}, c_{1}, \ldots, c_{n}\right]}\left(T_{\vec{c}}^{d}\right)^{*} C T_{\vec{c}}^{d}=\operatorname{rank}_{\mathbb{C}\left[c_{1}, \ldots, c_{n}\right]} D^{*} T_{\vec{c}}^{d}
$$

Applying Lemma 3.5 again, we get

$$
k_{1} \leq K_{n}(k) .
$$

Thus rank $r \leq K_{n}\left(K_{n}(k)\right)$, and we have an upper bound for rank $r$ that is independent of degree. We complete the proof by defining $R_{n}(k)$ to be the maximum rank $r$ for any bihomogeneous polynomial $r$ with $\left.\max _{H} \operatorname{rank} r\right|_{H} \leq k$.

Remark 4.2. In the proof of Lemma 4.1, we proved that

$$
R_{n}(k) \leq K_{n}\left(K_{n}(k)\right) .
$$

In fact, we believe that $R_{n}(k)=K_{n}(k)$.

We are now ready to prove the main result, Theorem 1.2 . For reader convenience we restate the theorem.

Theorem. Let $n \geq 2, z \in \mathbb{C}^{n+1}$, and $1 \leq m \leq n-1$. Then there exists a function $R_{m, n}: \mathbb{N}_{0} \rightarrow \mathbb{N}_{0}$ such that for any bihomogeneous polynomial $r(z, \bar{z})$,

$$
\operatorname{rank} r \leq R_{m, n}\left(\left.\max _{L \in G(m+1, n+1)} \operatorname{rank} r\right|_{L}\right) .
$$

Proof. Suppose $r$ is a nonzero bihomogeneous polynomial of bidegree $(d, d)$. We again write $r(z, \bar{z})=\left\langle C \mathcal{Z}_{n, d}, \mathcal{Z}_{n, d}\right\rangle$. After applying a linear change of coordinates we assume without loss of generality that all $L \in G(m+1, n+1)$ are given as

$$
z^{\prime}=V z^{\prime \prime},
$$

for $\left(z^{\prime}, z^{\prime \prime}\right) \in \mathbb{C}^{n-m} \times \mathbb{C}^{m+1}$ and $V \in M_{n-m, m+1}$ is a matrix. Using the embedding $z^{\prime \prime} \mapsto$ $\left(V z^{\prime \prime}, z^{\prime \prime}\right)$ we obtain a restriction matrix $T_{L}$. We notice that $T_{L}$ depends holomorphically on the entries of $V$.

We write

And again note that

$$
\left.r\right|_{L}(z, \bar{z})=\left\langle T_{L}^{*} C T_{L} \mathcal{Z}_{m, d}, \mathcal{Z}_{m, d}\right\rangle
$$

$$
\left.\operatorname{rank} r\right|_{L}=\operatorname{rank} T_{L}^{*} C T_{L}
$$


If $m=n-1$ we are done. We let $R_{n-1, n}(k)=R_{n}(k)$. Otherwise, $T_{L}$ is a composition $T_{L}=T_{S} T_{H}$ where $H$ is a hyperplane through the origin in $S$, where $S$ is an $m+2$ dimensional plane through the origin in $\mathbb{C}^{n+1}$. As $T_{L}^{*} C T_{L}=\left(T_{H} T_{S}\right)^{*} C T_{S} T_{H}$ is of rank less than $k, T_{S}^{*} C T_{S}$ is of rank less than $R_{m+1}(k)$ by Lemma 4.1. The theorem follows by applying Lemma 4.1 $(n-m)$ times. The function $R_{m, n}(k)$ is then defined recursively by composition.

We say a set $\mathcal{L} \subset G(m+1, n+1)$ is generic if $\mathcal{L}$ is not contained in any proper complex algebraic subvariety of $G(m+1, n+1)$. That is, no nontrivial polynomial vanishes on $\mathcal{L}$. With this terminology, we have the following corollary:

Corollary 4.3. Let $n \geq 2, z \in \mathbb{C}^{n+1}$, and $1 \leq m \leq n-1$. There exists a function $K_{m, n}: \mathbb{N}_{0} \rightarrow \mathbb{N}_{0}$ such that for any positive semidefinite bihomogeneous polynomial $r(z, \bar{z})$, and a generic subset $\mathcal{L} \subset G(m+1, n+1)$, we have

$$
\operatorname{rank} r \leq K_{m, n}\left(\left.\max _{L \in \mathcal{L}} \operatorname{rank} r\right|_{L}\right) .
$$

Note that $r(z, \bar{z}) \geq 0$ for all $z \in \mathbb{C}^{n+1}$ is not enough to conclude that the matrix of coefficients is positive semidefinite. On the other hand, if the matrix is positive semidefinite, then $r(z, \bar{z}) \geq 0$ is true for all $z \in \mathbb{C}^{n+1}$. See $[8,9]$ for more on positivity conditions.

Proof. The proof carries on as before after we make the following observation. Let $r(z, \bar{z})=$ $\left\langle C \mathcal{Z}_{n, d}, \mathcal{Z}_{n, d}\right\rangle$. If $C$ is positive semidefinite then we write $C=A^{*} A$. The restriction of $r$ to $L$ is

$$
\left.r\right|_{L}(z, \bar{z})=\left\langle T_{L}^{*} C T_{L} \mathcal{Z}_{m, d}, \mathcal{Z}_{m, d}\right\rangle
$$

Again note that

$$
\left.\operatorname{rank} r\right|_{L}=\operatorname{rank} T_{L}^{*} C T_{L}=\operatorname{rank} A T_{L}
$$

Let $k=\left.\max _{L \in \mathcal{L}} \operatorname{rank} r\right|_{L}$. The condition

$$
\operatorname{rank} A T_{L} \leq k
$$

is defined by the vanishing of certain polynomials in the entries of $A T_{L}$. Defining $L$ with a matrix $V \in M_{n-m, m+1}$ as in (41), we notice that as $A$ is fixed, then (47) is defined by vanishing of certain polynomials in the entries of $V$. We know that (47) is true for all $L \in \mathcal{L}$, where $\mathcal{L}$ is not contained in any complex algebraic subvariety of $G(m+1, n+1)$. The set of corresponding matrices $V$ is not contained in any complex algebraic subvariety of $M_{n-m, m+1}$. Thus (47) holds for all $V \in M_{n-m, m+1}$, and so for all $L \in G(m+1, n+1)$.

The conclusion follows by applying Lemma $3.5(n-m)$ times. The function $K_{m, n}(k)$ is then defined recursively by composition as in the proof of Theorem 1.2 .

\section{REAL-ANALYTIC CASE}

We wish to decompose real-analytic functions as a difference of squared norms of Hilbert space valued holomorphic functions. This line of reasoning follows the ideas pioneered by D'Angelo [5]. We introduce a rescaling that allows us to work with bounded operators.

First we prove that the matrix of coefficients is a trace-class operator if the series converges in the right neighborhood. See the book [1] for more information on matrix operators on $\ell^{2}$. We think of $\mathbb{C}^{n}$ as an open subset of $\mathbb{C P}^{n}$ by setting $z_{0}=1$. Therefore in $\mathbb{C}^{n}$ our coordinates will be $z=\left(z_{1}, \ldots, z_{n}\right) \in \mathbb{C}^{n}$. Let $\mathcal{Z}=\mathcal{Z}_{n}=\left(\ldots, z^{\alpha}, \ldots\right)$ be the mapping of $z \in \mathbb{C}^{n}$ to the space of infinite sequences, where components of the mapping are all possible monomials. By using the geometric series we note that $\mathcal{Z}$ maps the unit polydisc $\Delta$ to $\ell^{2}$. 
Lemma 5.1. Let $\Delta \subset \mathbb{C}^{n}$ be the unit polydisc. Suppose that $r(z, \bar{z})$ is a real-analytic function whose complexified power series at 0 converges in a neighborhood of $\bar{\Delta} \times \bar{\Delta} \subset \mathbb{C}^{n} \times \mathbb{C}^{n}$. Then the matrix of coefficients of $r$ defines a trace-class operator on $\ell^{2}$.

Proof. Write $r$ as $\langle C \mathcal{Z}, \mathcal{Z}\rangle$ where $C=\left[c_{\alpha \beta}\right]_{\alpha \beta}$ is the matrix of coefficients. The hypothesis says that the interior of the domain of convergence includes the point $z=(1,1, \ldots, 1)$. As the series converges absolutely at $(1,1, \ldots, 1)$ we get $\sum_{\alpha \beta}\left|c_{\alpha \beta}\right|<\infty$. A matrix whose entries are summable defines a compact operator. Moreover, if $\left\{e_{\alpha}\right\}$ is the standard basis corresponding to the monomials, then

$$
\sum_{\alpha}\left\langle|C| e_{\alpha}, e_{\alpha}\right\rangle \leq \sum_{\alpha}\left\||C| e_{\alpha}\right\|=\sum_{\alpha}\left\|C e_{\alpha}\right\| \leq \sum_{\alpha} \sum_{\beta}\left|\left\langle C e_{\alpha}, e_{\beta}\right\rangle\right|<\infty .
$$

Here $|C|$ is the unique positive Hermitian square root of $C^{*} C$. So $C$ is trace-class.

As $C$ is a Hermitian trace-class operator, we apply the spectral theorem. We write $C$ as a sum of rank one matrices as follows. Let $\lambda_{k}$ be the $k$-th nonzero eigenvalue. Let $\epsilon_{k}= \pm 1$ be the sign of the $k$-th nonzero eigenvalue. We ignore all zero eigenvalues. Let $\left\{v_{k}\right\}$ be an orthonormal set of corresponding eigenvectors. Let $m \in \mathbb{N}_{0} \cup\{\infty\}$ be the rank of $C$, then we write

$$
C=\sum_{j=1}^{m} \lambda_{j} v_{j} v_{j}^{*}
$$

The sum converges since the eigenvalues of a trace-class operator are absolutely summable. We define holomorphic functions $f_{j}(z)=\sqrt{\left|\lambda_{j}\right|} v_{j}^{*} \mathcal{Z}$. As $v_{j} \in \ell^{2}$, we note that each $f_{j}$ converges in the unit polydisc. We see that

$$
\langle C \mathcal{Z}, \mathcal{Z}\rangle=\sum_{j=1}^{m} \epsilon_{j}\left|f_{j}(z)\right|^{2} .
$$

Suppose that for some $m^{\prime}, \epsilon_{j}^{\prime}= \pm 1$, and some $g_{j}$ holomorphic in the unit polydisc we write

$$
\langle C \mathcal{Z}, \mathcal{Z}\rangle=\sum_{j=1}^{m^{\prime}} \epsilon_{j}^{\prime}\left|g_{j}(z)\right|^{2} .
$$

If $m^{\prime}=\infty$, then obviously $m^{\prime} \geq m$. If $m^{\prime}$ is finite then we have written $C$ as a sum of $m^{\prime}$ rank-one operators, and hence $m^{\prime} \geq m$.

Next we have to show that the rank of the matrix $C$ is invariant under change of coordinates. We define the rank of $r$ at the origin to be the rank of the matrix $C$.

Lemma 5.2. Let $\Delta \subset \mathbb{C}^{n}$ be the unit polydisc. Suppose that $r(z, \bar{z})$ is a real-analytic function whose complexified power series at 0 converges in a neighborhood of $\bar{\Delta} \times \bar{\Delta} \subset \mathbb{C}^{n} \times \mathbb{C}^{n}$.

Suppose that $\varphi$ is a biholomorphic change of coordinates that takes a neighborhood of $\bar{\Delta}$ to a neighborhood of $\bar{\Delta}$. Then ro $\varphi$ (which also converges on a neighborhood of $\bar{\Delta} \times \bar{\Delta} \subset \mathbb{C}^{n} \times \mathbb{C}^{n}$ ) has the same rank as $r$ at the origin.

Proof. Suppose that $r$ is of finite rank $k$. Then there exist $k$ eigenvectors of $C$, and therefore we write

$$
r(z, \bar{z})=\sum_{j=1}^{k} \epsilon_{j}\left|f_{j}(z)\right|^{2}
$$


for $\epsilon_{j}= \pm 1$ and $f_{j}$ holomorphic functions. Therefore

$$
(r \circ \varphi)(z, \bar{z})=\sum_{j=1}^{k} \epsilon_{j}\left|f_{j}(\varphi(z))\right|^{2},
$$

so rank $r \circ \varphi \leq \operatorname{rank} r$. We are finished by symmetry.

We define the number of positive and negative eigenvalues at a point (the rank of the positive or negative definite part) in the obvious way. Using exactly the same argument as above, we can show that the rank, the number of positive, and the number of negative eigenvalues are constant on connected sets.

Lemma 5.3. Let $U \subset \mathbb{C}^{n}$ be a connected open set. Let $r: U \rightarrow \mathbb{R}$ be a real-analytic function. Let $p_{1}, p_{2} \in U$, and let $k_{j}, a_{j}, b_{j}$ denote the rank, the number of positive, and the number of negative eigenvalues respectively at $p_{j}$. Then $k_{1}=k_{2}, a_{1}=a_{2}$, and $b_{1}=b_{2}$.

With the aid of the lemmas above we define the rank of $r$ regardless of where it converges. We simply translate and dilate $r$ so that it converges in the unit polydisc and then take the rank of the resulting compact operator.

If the domain $U$ of $r$ is connected, we define the rank of $r$ as the rank at some fixed point of $U$. Similarly define the signature pair to be the pair $(a, b)$ if $r$ has $a$ positive and $b$ negative eigenvalues. We allow $a$ and $b$ to be in $\mathbb{N}_{0} \cup\{\infty\}$.

Lemma 5.4. Let $L$ be an affine complex submanifold of $\mathbb{C}^{n}$ of dimension $m$ that intersects the unit polydisc $\Delta_{n} \subset \mathbb{C}^{n}$ and $E: \mathbb{C}^{m} \rightarrow L$ an embedding such that $E$ takes the closed unit polydisc $\bar{\Delta}_{m} \subset \mathbb{C}^{m}$ to a subset of $L \cap \Delta_{n}$.

The restriction matrix $T_{L}$ defined by $\mathcal{Z}_{n} \circ E=T_{L} \mathcal{Z}_{m}$ is a Hilbert-Schmidt operator.

Proof. Note that $\left\|\mathcal{Z}_{n}\right\|^{2}=\left\langle\mathcal{Z}_{n}, \mathcal{Z}_{n}\right\rangle$ is the absolute value squared of the geometric series and hence converges in the unit polydisc $\Delta_{n} \subset \mathbb{C}^{n}$. As $E$ takes the closed unit polydisc $\bar{\Delta}_{m} \subset \mathbb{C}^{m}$ to $\Delta_{n}$, we have that $\left\|\mathcal{Z}_{n} \circ E\right\|^{2}$ converges absolutely for $z \in \bar{\Delta}_{m}$, therefore

$$
\left\|\mathcal{Z}_{n} \circ E\right\|^{2}=\left\langle\mathcal{Z}_{n} \circ E, \mathcal{Z}_{n} \circ E\right\rangle=\left\langle T_{L}^{*} T_{L} \mathcal{Z}_{m}, \mathcal{Z}_{m}\right\rangle
$$

where $T_{L}$ is the restriction matrix induced by the embedding $E$. By Lemma 5.1, $T_{L}^{*} T_{L}$ is trace-class and so $T_{L}$ is a Hilbert-Schmidt operator.

When $r(z, \bar{z})=\langle C \mathcal{Z}, \mathcal{Z}\rangle$ is positive semi-definite, that is, has no negative eigenvalues, we write

$$
r(z, \bar{z})=\left\langle A^{*} A \mathcal{Z}, \mathcal{Z}\right\rangle=\|A \mathcal{Z}\|^{2} .
$$

When everything is scaled appropriately so that $C$ is trace-class, then $A$ is Hilbert-Schmidt.

Next we note that we can write a finite rank holomorphic mapping in a convenient way when working in general coordinates. The idea is very similar to the technique used to prove Green's theorem in [12. Of course now we are working in nonhomogeneous coordinates so we must make a generic choice of affine coordinates instead of linear. The following proposition allows us to prove our main result by reducing to the case where each component of $A \mathcal{Z}$ is a single monomial.

We use the same somewhat nonstandard reverse lexicographic order, or reverse lex, as in [12]. Reverse lex is the common reverse lex ordering in each degree, but we order degrees by putting the lower total degree first. For example when $n=3$ we have

$$
1>z_{1}>z_{2}>z_{3}>z_{1}^{2}>z_{1} z_{2}>z_{2}^{2}>z_{1} z_{3}>z_{2} z_{3}>z_{3}^{2}>\cdots
$$


When writing down the monomials, for example in $\mathcal{Z}$, we write then down in decreasing order as written above. In this ordering, the initial monomial of a series is the maximal monomial that appears. We call a set $\mathcal{M}$ of monomials affine-Borel-fixed if whenever $z^{\alpha} \in \mathcal{M}$ and $z_{j} \mid z^{\alpha}$, then $z^{\alpha} \frac{1}{z_{j}}$ and $z^{\alpha} \frac{z_{\ell}}{z_{j}}$ is in $\mathcal{M}$ for all $\ell<j$. If a finite affine-Borel-fixed set of monomials is homogenized to the same degree via a new variable $z_{0}$, where $z_{0}>z_{1}$, then the set is Borel-fixed in the standard sense (that is, the affine step becomes same as the others). As usual by pivot columns we mean the columns of the leading terms after row reduction. The following proposition is an analogue of Galligo's Theorem (see Theorem 1.27 in [12]) to our setting. The proof technique seems standard, but we include it for completeness.

Proposition 5.5. Let $f(z)=A \mathcal{Z}$ be a finite rank holomorphic mapping, with linearly independent components, from a neighborhood of the closed unit polydisc $\bar{\Delta} \subset \mathbb{C}^{n}$ to $\mathbb{C}^{N}$. That is, $A$ is an $N \times \infty$ matrix of rank $N$. Let $\mathcal{Z}$ be given in the monomial order as above (or any multiplicative monomial order). Then there exists an affine self mapping $\chi$ of $\mathbb{C}^{n}$ arbitrarily close to the identity, and an $N \times N$ invertible matrix $B$ such that

$$
B A \mathcal{Z} \circ \chi=\widetilde{A} \mathcal{Z} .
$$

where $\widetilde{A}$ is a rank $N$ matrix in reduced row echelon form such that the pivot columns correspond to an affine-Borel-fixed set of monomials. Furthermore, the set of such $\chi$ is an open dense subset of a neighborhood of the identity in the space of affine maps.

Proof. Let $A_{p}$ denote the first $p$ columns of the matrix $A$ followed by zeros. Let $A^{\chi}$ be defined by $A^{\chi} \mathcal{Z}=A \mathcal{Z} \circ \chi$, for affine maps $\chi$. Because rank is given by looking at certain subdeterminants, for a fixed $p$ the rank of $\left(A^{\chi}\right)_{p}$ is maximal and constant on a Zariski open set in a neighborhood of the identity in the space of affine maps $\chi$. There are only finitely many $p$ for which the rank of $\left(A^{\chi}\right)_{p}$ is less than $N$. The intersection of finitely many Zariski open sets is Zariski open. Therefore, after applying an affine map close to the identity we can assume that for each $p$, the rank of $\left(A^{\chi}\right)_{p}$ is maximal (constant) for all $\chi$ in a neighborhood of the identity.

Take $q$ large enough such that $A_{q}$ is rank $N$. The ranks of all submatrices of $\left(A^{\chi}\right)_{q}$ achieve a maximum on a Zariski open set, and therefore we assume that the ranks of all submatrices of $\left(A^{\chi}\right)_{q}$ are constant for all $\chi$ near the identity.

The $p$ where the rank of $A_{p}$ increases are precisely the columns with the pivot elements. We will show that these columns are affine-Borel-fixed.

Suppose for contradiction that $p$ is the first pivot column that violates the affine-Borelfixed property in the following way. Without loss of generality suppose that $p$ corresponds to $z^{\alpha}$ where $z_{1} \mid z^{\alpha}$. And suppose that the $j$-th column $(j<p)$ corresponding to $z^{\alpha} \frac{1}{z_{1}}$ does not contain a pivot. The other moves mentioned above are similar, the map used below need not be affine but linear.

Write $Z=\left(z_{2}, \ldots, z_{n}\right)$, and let $\beta \in \mathbb{N}_{0}^{n-1}$ be a multi-index for $Z$ such that $\alpha=(m+1, \beta)$ for some $m \geq 0$. Therefore, by the fact that $\alpha$ is the first place where the affine-Borelfixed property is violated, we know that there exist pivots in columns corresponding to $(0, \beta),(1, \beta), \ldots,(m-1, \beta)$, and in $(m+1, \beta)$, but not at $(m, \beta)$. The pivot at $(m+1, \beta)$ corresponds to the last nonzero column in $A_{p}$.

Without loss of generality we assume that $\operatorname{rank} A_{p}=N$. This simplification is possible by taking all the pivot columns in $A_{p}$ and in each one picking a pivot element that we would have used in the row reduction, and then disregarding all rows without a pivot element. The 
same pivot elements can be used after the simplification and therefore the location of pivot columns in $A_{p}$ is unchanged.

Let $\gamma \in \mathbb{N}_{0}^{n-1}$ run over the multi-indices for $Z$. We write $f$ as

$$
f(z)=f\left(z_{1}, Z\right)=\sum_{j, \gamma} w_{\gamma}^{j} z_{1}^{j} Z^{\gamma}
$$

for $N$-vectors $w_{\gamma}^{j}$. Take the affine map that replaces $z_{1}$ with $z_{1}+c$. We then have

$$
f\left(z_{1}+c, Z\right)=\sum_{j, \gamma}\left(\sum_{\ell=0}^{\infty} c^{\ell}\left(\begin{array}{c}
j+\ell \\
j
\end{array}\right) w_{\gamma}^{j+\ell}\right) z_{1}^{j} Z^{\gamma} .
$$

Let the multi-indices $\left(k_{1}, \gamma_{1}\right), \ldots,\left(k_{s}, \gamma_{s}\right)$, where $k_{j} \in \mathbb{N}_{0}$ and $\gamma_{j} \in \mathbb{N}_{0}^{n-1}, \gamma_{j} \neq \beta$, correspond to the remaining pivot columns in $A_{p}$. Note $s+m+1=N$. Because $A_{p-1}$ is of rank lower than $N=\operatorname{rank} A_{p}$, we have $\operatorname{det}\left(w_{\beta}^{0}, \ldots, w_{\beta}^{m}, w_{\gamma_{1}}^{k_{1}}, \ldots, w_{\gamma_{s}}^{k_{s}}\right)=0$. For simplicity, in the next calculation we write $W_{1}=\left[w_{\beta}^{0}, \ldots, w_{\beta}^{m}\right]$ and $W_{2}=\left[w_{\gamma_{1}}^{k_{1}}, \ldots, w_{\gamma_{s}}^{k_{s}}\right]$. We take the determinant of the same columns after applying our affine map:

$$
\begin{aligned}
& \operatorname{det}\left(\sum_{\ell=0}^{\infty} c^{\ell}\left(\begin{array}{c}
0+\ell \\
0
\end{array}\right) w_{\beta}^{0+\ell}, \ldots, \sum_{\ell=0}^{\infty} c^{\ell}\left(\begin{array}{c}
m+\ell \\
m
\end{array}\right) w_{\beta}^{m+\ell},\right. \\
& \left.\quad \sum_{\ell=0}^{\infty} c^{\ell}\left(\begin{array}{c}
k_{1}+\ell \\
k_{1}
\end{array}\right) w_{\gamma_{1}}^{k_{1}+\ell}, \ldots, \sum_{\ell=0}^{\infty} c^{\ell}\left(\begin{array}{c}
k_{s}+\ell \\
k_{s}
\end{array}\right) w_{\gamma_{s}}^{k_{s}+\ell}\right) \\
& =\operatorname{det}\left(W_{1}, W_{2}\right)+c\left(\left(\begin{array}{c}
0+1 \\
0
\end{array}\right) \operatorname{det}\left(w_{\beta}^{0+1}, w_{\beta}^{1}, \ldots, w_{\beta}^{m}, W_{2}\right)\right. \\
& +\left(\begin{array}{c}
1+1 \\
1
\end{array}\right) \operatorname{det}\left(w_{\beta}^{0}, w_{\beta}^{1+1}, \ldots, w_{\beta}^{m}, W_{2}\right)+\cdots+\left(\begin{array}{c}
m+1 \\
m
\end{array}\right) \operatorname{det}\left(w_{\beta}^{0}, w_{\beta}^{1}, \ldots, w_{\beta}^{m+1}, W_{2}\right) \\
& \left.+\left(\begin{array}{c}
k_{1}+1 \\
k_{1}
\end{array}\right) \operatorname{det}\left(W_{1}, w_{\gamma_{1}}^{k_{1}+1}, \ldots, w_{\gamma_{s}}^{k_{s}}\right)+\cdots+\left(\begin{array}{c}
k_{s}+1 \\
k_{s}
\end{array}\right) \operatorname{det}\left(W_{1}, w_{\gamma_{1}}^{k_{1}}, \ldots, w_{\gamma_{s}}^{k_{s}+1}\right)\right)
\end{aligned}
$$

By assumption the ranks are constant for all $c$ near zero and so this function must be identically zero. In particular the coefficient of $c$ must be zero:

$$
\begin{aligned}
& \operatorname{det}\left(w_{\beta}^{0+1}, w_{\beta}^{1}, \ldots,\right.\left.w_{\beta}^{m}, w_{\gamma_{1}}^{k_{1}}, \ldots, w_{\gamma_{s}}^{k_{s}}\right)+2 \operatorname{det}\left(w_{\beta}^{0}, w_{\beta}^{1+1}, \ldots, w_{\beta}^{m}, w_{\gamma_{1}}^{k_{1}}, \ldots, w_{\gamma_{s}}^{k_{s}}\right) \\
&+\cdots+m \operatorname{det}\left(w_{\beta}^{0}, w_{\beta}^{1}, \ldots, w_{\beta}^{(m-1)+1}, w_{\beta}^{m}, w_{\gamma_{1}}^{k_{1}}, \ldots, w_{\gamma_{s}}^{k_{s}}\right) \\
&+(m+1) \operatorname{det}\left(w_{\beta}^{0}, w_{\beta}^{1}, \ldots, w_{\beta}^{m-1}, w_{\beta}^{m+1}, w_{\gamma_{1}}^{k_{1}}, \ldots, w_{\gamma_{s}}^{k_{s}}\right) \\
&+\left(k_{1}+1\right) \operatorname{det}\left(w_{\beta}^{0}, \ldots, w_{\beta}^{m}, w_{\gamma_{1}}^{k_{1}+1}, \ldots, w_{\gamma_{s}}^{k_{s}}\right)+\cdots+\left(k_{s}+1\right) \operatorname{det}\left(w_{\beta}^{0}, \ldots, w_{\beta}^{m}, w_{\gamma_{1}}^{k_{1}}, \ldots, w_{\gamma_{s}}^{k_{s}+1}\right)=0 .
\end{aligned}
$$

The first $m$ determinants are trivially zero as there are repeated columns. We next show that for all $j=1, \ldots, s$ we get $\operatorname{det}\left(w_{\beta}^{0}, \ldots, w_{\beta}^{m}, w_{\gamma_{1}}^{k_{1}}, \ldots, w_{\gamma_{j}}^{k_{j}+1}, \ldots, w_{\gamma_{s}}^{k_{s}}\right)=0$. Consider two cases. First suppose that in the monomial ordering $\left(k_{j}, \gamma_{j}\right)>(m, \beta)$. As the columns of $A$ are sorted in decreasing order, the column for $\left(k_{j}, \gamma_{j}\right)$ is to the left of the column for $(m, \beta)$. The ordering is multiplicative, and so $\left(k_{j}+1, \gamma_{j}\right)>(m+1, \beta)$. If $w_{\gamma_{j}}^{k_{j}+1}$ is a pivot column, 
then it must be equal to $w_{\gamma_{i}}^{k_{i}}$ for some $i \neq j$, and the determinant must be zero. So suppose that $w_{\gamma_{j}}^{k_{j}+1}$ is not a pivot column. As both $w_{\beta}^{m}$ and $w_{\gamma_{j}}^{k_{j}+1}$ are not pivot columns, they are linear combinations of the preceeding columns. In particular $w_{\beta}^{m+1}$ is not a vector in the linear combination for either $w_{\beta}^{m}$ or $w_{\gamma_{j}}^{k_{j}+1}$. Therefore the determinant must be 0 .

Let us next suppose $(m, \beta)>\left(k_{j}, \gamma_{j}\right)$. We write $w_{\beta}^{m}$ as a linear combination of preceeding columns, and note that the linear combination includes neither $w_{\beta}^{m+1}$ nor $w_{\gamma_{j}}^{k_{j}}$. Again this implies that the determinant must be zero.

Therefore,

$$
\operatorname{det}\left(w_{\beta}^{0}, w_{\beta}^{1}, \ldots, w_{\beta}^{m-1}, w_{\beta}^{m+1}, w_{\gamma_{1}}^{k_{1}}, \ldots, w_{\gamma_{s}}^{k_{s}}\right)=0 .
$$

This is a contradiction as $w_{\beta}^{m+1}$ is the $p$ th column that we assumed to have a pivot entry. These are all the pivot entries and the matrix $\left[w_{\beta}^{0}, w_{\beta}^{1}, \ldots, w_{\beta}^{m-1}, w_{\beta}^{m+1}, w_{\gamma_{1}}^{k_{1}}, \ldots, w_{\gamma_{s}}^{k_{s}}\right]$ has $N$ pivots and the determinant should be nonzero.

The other non-affine moves are similar, but instead of $z_{i}+c$ we take $z_{i}+c z_{j}$ where $j<i$. We finish by row reducing the coefficient matrix.

We now prove the real-analytic version of the main theorem on rank of Hermitian forms for positive semi-definite forms. The proof reduces to the algebraic version. However, note that a key point here is that the rank of $r$ is not required to be finite. As before a generic subset $\mathcal{L} \subset G_{m, n}$ is a subset not contained in any proper complex algebraic subvariety of $G_{m, n}$, that is, no nontrivial polynomial vanishes on $\mathcal{L}$.

Lemma 5.6. Let $n \geq 2$ and $1 \leq m \leq n-1$. There exists a function $K_{m, n}: \mathbb{N}_{0} \rightarrow \mathbb{N}_{0}$ with the following property. For any $r(z, \bar{z})$ positive semi-definite real-analytic function whose complexified power series at 0 converges in a neighborhood of $\bar{\Delta} \times \bar{\Delta} \subset \mathbb{C}^{n} \times \mathbb{C}^{n}$, and $\mathcal{L} \subset G_{m, n}$ a generic subset so that all the corresponding manifolds intersect $\Delta$ and

$$
\left.\sup _{L \in \mathcal{L}} \operatorname{rank} r\right|_{L}<\infty
$$

Then rank $r$ is finite and moreover

$$
\operatorname{rank} r \leq K_{m, n}\left(\left.\max _{L \in \mathcal{L}} \operatorname{rank} r\right|_{L}\right) .
$$

Proof. When referring to the homogeneous polynomial results of sections 2 and 3 , let us keep in mind that we are thinking of $\mathbb{C}^{n}$ as an open subset of $\mathbb{C P}^{n}$ via setting $z_{0}=1$, and hence here our variables are $z=\left(z_{1}, \ldots, z_{n}\right) \in \mathbb{C}^{n}$.

For an affine manifold $L$ that passes through the unit polydisc, we find an embedding $E: \mathbb{C}^{m} \rightarrow H$ such that $E$ takes the closed unit polydisc $\bar{\Delta}_{m} \subset \mathbb{C}^{m}$ to a subset of $H \cap \Delta_{n}$. The induced restriction matrix $T_{L}$ is a Hilbert-Schmidt operator by Lemma 5.4.

As before, we assume that $L$ is a graph over the first $m$ variables and we assume that it contains a point of the set $\left\{z: z_{1}=z_{2}=\cdots=z_{m}=0,\left|z_{j}\right|<1\right.$ for $\left.j=m+1, \ldots, n\right\}$. So assume that $\mathcal{L}$ contains only such $L$. As in the proof of Theorem 1.2 we identify $\mathcal{L}$ with a subset $\mathcal{V} \subset M_{n-m, m+1}$. For such $L$ we take a dilation of the first $m$ variables to obtain an embedding $E$ that maps the closed unit polydisc $\bar{\Delta}_{m}$ into $\Delta_{n}$, to ensure that $T_{L}$ is a Hilbert-Schmidt operator. Without loss of generality, we also assume that the dilation is the same for all $L \in \mathcal{L}$. Therefore we again assume that entries of $T_{L}$ depend holomorphically on the entries of $V \in \mathcal{V}$. 
Let $k=\left.\max _{L \in \mathcal{L}} \operatorname{rank} r\right|_{L}$. Write

$$
r(z, \bar{z})=\left\langle A^{*} A \mathcal{Z}, \mathcal{Z}\right\rangle
$$

Thus for $L \in \mathcal{L}$ we have

$$
\operatorname{rank} A T_{L} \leq k .
$$

As for the finite case, this condition is defined by the vanishing of polynomials in the entries of $A T_{L}$, these depend holomorphically on the entries of $V$. Thus (65) is true for all affine manifolds of dimension $m$ that intersect $\Delta$.

Unless $m=n-1$ we again write $T_{L}=T_{S} T_{H}$ for a hyperplane $H$ in $S$, where $S$ is an affine manifold of dimension $m+1$. Hence if we prove the result of the theorem for hyperplanes, the general case follows by induction as before.

Therefore assume that

$$
\left.\operatorname{rank} r\right|_{H} \leq k
$$

for all affine hyperplanes $H$ that intersect $\Delta$. Then we have that $\operatorname{rank} A T_{H} \leq k$ for all such hyperplanes $H$.

We first assume that $A$ is finite rank. The maximum rank of $\left.r\right|_{H}$ is achieved on a generic affine hyperplane. After a generic affine change of variables we assume that $H$ is given by $\left\{z_{n}=0\right\}$. Let $\mathcal{Z}$ be in the reverse lex monomial order. Using Proposition 5.5 we assume also that $A$ is in row reduced echelon form and the pivot columns of $A$ are affine-Borel-fixed. If we take the identity embedding of our hyperplane, then the restriction matrix is simply a matrix $T_{H}$ with zeros everywhere except a 1 in each column in the row corresponding to monomials not depending on $z_{n}$. That is, $A T_{H}$ contains those columns of $A$ that correspond to monomials that do not depend on $z_{n}$. We compute

$$
\left.\operatorname{rank} r\right|_{H}=\operatorname{rank} A T_{H} \geq \operatorname{rank} P T_{H},
$$

where $P$ is the matrix with just the pivot columns from $A$ left and all other columns set to 0 . The matrix $P$ is a matrix for a polynomial of degree $d$, therefore we now truncate the series, homogenize with $z_{0}$ and order the monomials in $\mathcal{Z}_{n, d}$ according to reverse lex ordering. Let us call $P^{\prime}$ this new finite matrix that only goes up to degree $d$, and $T_{H}^{\prime}$ the new truncated finite restriction matrix for up to degree $d$. The pivot columns in $P^{\prime}$ (the only columns with nonzero entries) are still of course Borel-fixed. Therefore, a generic small linear change of coordinates in $\mathbb{C}^{n+1}$ does not change the pivot columns. Furthermore, since all monomials are now homogeneous of degree $d$, and in reverse lex ordering, once a monomial is divisible by $z_{n}$ all the monomials to the right are also divisible by $z_{n}$. Therefore, a small linear change of coordinates does not change the rank of the restriction to $H=\left\{z_{n}=0\right\}$. We apply Lemma 3.5 to obtain the desired bound

$$
\operatorname{rank} r=N=\operatorname{rank} P^{\prime} \leq K_{n}\left(\operatorname{rank} P^{\prime} T_{H}^{\prime}\right) \leq K_{n}\left(\left.\operatorname{rank} r\right|_{H}\right)
$$

Now let us drop the assumption that the rank of $A$ is finite. Let $A_{\nu}$ be an infinite by infinite matrix consisting of the first $\nu$ rows of $A$ followed by all zero rows. Obviously $A_{\nu}$ is of finite rank. We have that

$$
\operatorname{rank} A_{\nu} T_{H} \leq \operatorname{rank} A T_{H} \leq k .
$$

It follows that rank $A_{\nu} \leq K_{n}(k)$ for all $\nu$. 
From the proof of Lemma 5.1 we note that the entries of $A^{*} A$ are absolutely summable and hence the entries of $A$ are square summable. Therefore $A_{\nu}$ converges to $A$ in the HilbertSchmidt norm. Then as the rank of $A_{\nu}$ is uniformly bounded by $K_{n}(k)$, we obtain

$$
\operatorname{rank} A \leq K_{n}(k) .
$$

To see this fact, simply pick a finite orthonormal set $\left\{A w_{j}\right\}$ in the range of $A$ and then notice that $A_{\nu} w_{j}$ must converge to $A w_{j}$ as $\nu \rightarrow \infty$. Thus for large enough $\nu$, the set $\left\{A_{\nu} w_{j}\right\}$ must be linearly independent (and hence of cardinality less than $K_{n}(k)$ ).

Remark 5.7. We have already done quite a bit of the heavy lifting required to prove Lemma 3.5 outright. To finish the proof we must find the maximal size of an affine-Borel-fixed set of monomials such that at most $k$ of them do not depend on $z_{n}$. Precisely this combinatorics is done in [12]. The bound $K_{n}(k)$ is then the maximum size of such a set of monomials. It is instructive to see how the bound $K_{2}(k)=\frac{k(k+1)}{2}$ when $n=2$ can be obtained. When we restrict to $z_{2}=0$, then in each degree $d$, only one monomial out of at most $d+1$ survives.

We use this formulation of $K_{n}(k)$ to obtain a simpler bound on its size. We look at how $K_{n}(k)$ increases as $k$ increases. The largest increase is when we are allowed to take all monomials that depend on $z_{n}$ up to degree $d$. Thus,

$$
K_{n}\left(\left(\begin{array}{c}
n+d-1 \\
n-1
\end{array}\right)\right)=\left(\begin{array}{c}
n+d \\
n
\end{array}\right)=\left(1+\frac{d}{n}\right)\left(\begin{array}{c}
n+d-1 \\
n-1
\end{array}\right) .
$$

As $K_{n}(k)$ grows most when $k=\left(\begin{array}{c}n+d-1 \\ n-1\end{array}\right)$, we obtain a general bound for all $k$ by looking at these specific $k$.

Let $k=\left(\begin{array}{c}n+d-1 \\ n-1\end{array}\right)$. Using the crude estimate $k \geq\left(\frac{n+d-1}{n-1}\right)^{n-1}$, we get

$$
(n-1) k^{1 /(n-1)}-n+1 \geq d,
$$

and therefore

$$
K_{n}(k) \leq k\left(1+\frac{(n-1) k^{1 /(n-1)}-n+1}{n}\right)=\frac{n-1}{n} k^{\frac{n}{n-1}}+\frac{1}{n} k .
$$

Example 5.8. Let us show that positivity of $r$ is necessary. First note that the set $\mathcal{L}$ of affine complex manifolds of dimension 1 that lie in

$$
Q(2,1)=\left\{z \in \mathbb{C}^{3}:\left|z_{1}\right|^{2}+\left|z_{2}\right|^{2}-\left|z_{3}\right|^{2}=1\right\}
$$

forms a generic set in $G_{1,3}$. See section 6 for a proof of this fact. Let

$$
r(z, \bar{z})=\left(\left|z_{1}\right|^{2}+\left|z_{2}\right|^{2}-\left|z_{3}\right|^{2}\right)^{d} .
$$

Then for $z \in Q(2,1)$

$$
r(z, \bar{z})=\left(\left|z_{1}\right|^{2}+\left|z_{2}\right|^{2}-\left|z_{3}\right|^{2}\right)^{d}=1 .
$$

Therefore the rank of $\left.r\right|_{L}$ for $L \in \mathcal{L}$ is always 1 . However the rank of $r$ is $\left(\begin{array}{c}2+d \\ d\end{array}\right)$.

For an infinite rank example, note that $e^{r}$ is of infinite rank but the rank of $\left.e^{r}\right|_{L}$ is 1 .

We can now prove Theorem 1.1. That is, we can drop positivity of $r$ if we assume that the rank is bounded on all hyperplanes. The proof is very similar to the proof of Lemma 4.1 .

Proof of Theorem 1.1. Suppose $r$ is a nonzero Hermitian form. We assume that $r$ converges in a neighborhood of the closed unit polydisc as above. Write $r(z, \bar{z})=\left\langle C \mathcal{Z}_{n}, \mathcal{Z}_{n}\right\rangle$. It is 
again enough to prove the theorem for hyperplanes. Restricting $r$ to a hyperplane $H$ that intersects the closed unit polydisc, we have

$$
\left.r\right|_{H}(z, \bar{z})=\left\langle T_{H}^{*} C T_{H} \mathcal{Z}_{n-1}, \mathcal{Z}_{n-1}\right\rangle .
$$

Let $k=\left.\max _{H} \operatorname{rank} r\right|_{H}=\max _{H} \operatorname{rank} T_{H}^{*} C T_{H}$ where $H$ ranges over all complex hyperplanes. As before, the set of hyperplanes $H_{\vec{c}}$, defined by $1=c_{1} z_{1}+\cdots+c_{n} z_{n}$, is open and dense in the set of all hyperplanes. For an open set $U \subset \mathbb{C}^{n}$ of $\vec{c}$ giving hyperplanes that intersect the unit polydisc, we take an embedding, which depends holomorphically on $\vec{c}$, that takes the the closed unit polydisc into the unit polydisc. In what follows, $\vec{c}$ will vary over $U$.

$$
k=\left.\max _{\vec{c} \in U} \operatorname{rank} r\right|_{H_{\vec{c}}}=\max _{\vec{c}} \operatorname{rank}\left(T_{\vec{c}}^{d}\right)^{*} C T_{\vec{c}}^{d}=\max _{\bar{c}_{1}, \ldots, \bar{c}_{n}, c_{1}, \ldots, c_{n}} \operatorname{rank}\left(T_{\vec{c}}^{d}\right)^{*} C T_{\vec{c}}^{d} .
$$

Rank is defined by vanishing of certain subdeterminants. By polarizing we note that we can treat $c_{1}, \ldots, c_{n}$ and $\bar{c}_{1}, \ldots, \bar{c}_{n}$ as separate variables in the second maximum above.

Let $D=C T_{\vec{c}}^{d}, m=\operatorname{rank} C$, and let $k_{1}=\max _{c_{1}, \ldots, c_{n}} \operatorname{rank} D$. Note that we still allow the ranks to be infinite. Applying the bound (70) from the proof of Lemma 5.6 we obtain

$$
m \leq K_{n}\left(k_{1}\right) .
$$

For a generic choice of $c_{1}, \ldots, c_{n}$, we have

$$
k_{1}=\operatorname{rank} D=\operatorname{rank} D^{*} .
$$

Likewise, taking generic choices of $\bar{c}_{1}, \ldots, \bar{c}_{n}$, we have

$$
k=\max _{\bar{c}_{1}, \ldots, \bar{c}_{n}, b_{1}, \ldots, b_{n}} \operatorname{rank}\left(T_{\vec{c}}\right)^{*} C T_{\vec{b}}=\max _{b_{1}, \ldots, b_{n}} \operatorname{rank} D^{*} T_{\vec{b}} .
$$

Again applying the bound 70 we get

$$
k_{1} \leq K_{n}(k) .
$$

As $k$ is finite, then $k_{1}$ is finite and hence $m \leq K_{n}\left(K_{n}(k)\right)$. Defining $R_{n}(k)$ as in the proof of Lemma 4.1 completes the proof.

\section{CR MAPPINGS OF HYPERQUADRICS}

In this section we apply Theorem 1.1 on bounding the rank of Hermitian forms by the rank of restrictions to prove Theorem 1.3 on rigidity of CR mappings between hyperquadrics.

First let us note how to construct CR mappings of hyperquadrics from a real-analytic function $r(z, \bar{z})$ vanishing on the hyperquadric.

Proposition 6.1. Let $p \in Q(a, b)$ be a point and let $\Omega \subset \mathbb{C}^{a+b}$ be a connected open set with $p \in \Omega$. Let $r: \Omega \rightarrow \mathbb{R}$ be nonzero real-analytic function of finite rank with $A$ positive and $B$ negative eigenvalues such that $r$ vanishes on $Q(a, b) \cap \Omega$.

Then there exists a point $q$ arbitrarily close to $p$ and a neighborhood $U \subset Q(a, b)$ of $q$, and a real-analytic $C R$ mapping $F: U \rightarrow Q(A, B-1)$ whose image is not contained in a complex hyperplane.

Proof. Write

$$
r(z, \bar{z})=\sum_{j=1}^{A}\left|f_{j}(z)\right|^{2}-\sum_{j=1}^{B}\left|g_{j}(z)\right|^{2}
$$


with $f_{j}$ and $g_{j}$ linearly independent. The mapping

$$
z \mapsto\left(\frac{f_{1}(z)}{g_{B}(z)}, \ldots, \frac{f_{A}(z)}{g_{B}(z)}, \frac{g_{1}(z)}{g_{B}(z)}, \ldots, \frac{g_{B-1}(z)}{g_{B}(z)}\right)
$$

is a meromorphic mapping taking $Q(a, b)$ to $Q(A, B-1)$. If $g_{B}(p) \neq 0$, we are finished. Otherwise note that the set $\left\{g_{B}=0\right\} \cap Q(a, b)$ is nowhere dense in $Q(a, b)$ and hence there is a point $q \in Q(a, b)$ arbitrarily close where $g_{B}(q) \neq 0$ and a holomorphic mapping defined near $q$ taking $Q(a, b)$ to $Q(A, B-1)$.

Therefore if $r$ vanishes on $Q(a, b)$ and has signature pair $(A, B)$ then $r$ induces a mapping from $Q(a, b)$ to $Q(A, B-1)$.

Let us prove a lemma about signature pairs of functions vanishing on $Q(a, b)$ before proving Theorem 1.3 .

Lemma 6.2. Let $a \geq 2, b \geq 1$, and $a>b$. Let $\Omega \subset \mathbb{C}^{a+b}$ be a connected open set such that $\Omega \cap Q(a, b)$ is nonempty and $r: \Omega \rightarrow \mathbb{R}$ be a real-analytic function vanishing on $\Omega \cap Q(a, b)$ with signature pair $(A, B)$. If $B<\infty$ then

$$
A \leq M(a, b, B)
$$

where $M=M(a, b, B)$ is a constant depending only on $a, b$, and $B$.

Proof. Write

$$
r(z, \bar{z})=\sum_{j=1}^{A}\left|f_{j}(z)\right|^{2}-\sum_{j=1}^{B}\left|g_{j}(z)\right|^{2}=\|f(z)\|^{2}-\|g(z)\|^{2},
$$

such that the components of $f$ and $g$ are linearly independent. Let us suppose that $B$ is finite, but for now we allow $f$ to map into an infinite dimensional Hilbert space.

After an affine change of coordinates we assume that we are working in a neighborhood of the origin and that $f$ and $g$ converge in a neighborhood of a closed unit polydisc $\bar{\Delta}$. Let $\tilde{Q}(a, b)$ and $\tilde{\Omega}$ correspond to $Q(a, b)$ and $\Omega$ after this affine change of coordinates.

Let $\mathcal{Z}$ be the infinite vector of all monomials in $z$. We find an $A \times \infty$ matrix $F$ such that $f(z)=F \mathcal{Z}$. Similarly we have a $B \times \infty$ matrix $G$ such that $g(z)=G \mathcal{Z}$. We know that $\|f(z)\|^{2}$ converges in the closed unit polydisc, and so by Lemma $5.1 F$ defines a HilbertSchmidt operator $\left(F^{*} F\right.$ is trace-class) on $\ell^{2}$. Similarly $G$ defines a Hilbert-Schmidt operator.

For $z \in \tilde{Q}(a, b) \cap \tilde{\Omega}$ we have

$$
\|F \mathcal{Z}\|^{2}-\|G \mathcal{Z}\|^{2}=\|f(z)\|^{2}-\|g(z)\|^{2}=0 .
$$

As $f$ and $g$ are linearly independent we have

$$
\operatorname{rank} F=A \quad \text { and } \quad \operatorname{rank} G=B .
$$

Let $L$ be an affine complex submanifold of $\tilde{Q}(a, b)$. Taking the right embedding of $L$ and applying Lemma 5.4, we find that the restriction matrix $T_{L}$ is a Hilbert-Schmidt operator. Restricted to $L$ we have that

$$
\|F \mathcal{Z}\|^{2}=\|G \mathcal{Z}\|^{2}
$$

We thus have that $T_{L} F^{*} F T_{L}=T_{L} G^{*} G T_{L}$. In other words, the rank of $G T_{L}$ is equal to the rank of $F T_{L}$. The rank of $G T_{L}$ is at most $B$. That means that the rank of $F T_{L}$ is at most $B$ for all $L$ that lie in $\tilde{Q}(a, b)$. 
Let $\left(z^{\prime}, z^{\prime \prime}\right) \in \mathbb{C}^{a} \times \mathbb{C}^{b}$ be coordinates such that $Q(a, b)$ is defined by $\left\|z^{\prime}\right\|^{2}-\left\|z^{\prime \prime}\right\|^{2}=1$. We assume that $a>b$. The defining function for $L$ that lie in $Q(a, b)$ is

$$
z^{\prime}=V\left[\begin{array}{c}
z^{\prime \prime} \\
1
\end{array}\right],
$$

where $V \in M_{a, b+1}($ an $a \times(b+1)$ matrix $)$ has orthonormal columns. Let us denote by $L_{V}$ the affine manifold defined by such a $V$.

We claim that the set $\mathcal{V} \subset M_{a, b+1} \cong \mathbb{C}^{a(b+1)}$ of matrices with orthonormal columns is not contained in any proper complex algebraic subvariety of $\mathbb{C}^{a(b+1)}$. Suppose that there was a polynomial $p$ in $a(b+1)$ variables that vanishes on $\mathcal{V}$. If we multiply the $k$-th column by $e^{i \theta_{k}}$ we note that $p$ still has to vanish. By the uniqueness theorem for Fourier series, there has to be a polynomial that is independently homogeneous in entries of the $k$-th column (for all columns). That is, we can multiply each column independently by any complex number and still stay in the zero locus of $p$. The claim follows by working on each column independently.

The claim shows that the set $\mathcal{L}$ of affine manifolds $L$ corresponding to $V \in \mathcal{V}$ is a generic set in $G_{m, n}$. If we let $\tilde{\mathcal{L}} \subset G_{m, n}$ correspond to the set of affine manifolds that lie in $\tilde{Q}(a, b)$, we see that $\tilde{\mathcal{L}}$ must be generic as well.

We have all the ingredients to apply Lemma 5.6 to obtain that

$$
A=\operatorname{rank} F \leq K_{b, a+b}(B) .
$$

Let us restate Theorem 1.3 for reader convenience before proving it.

Theorem. Let $a>b \geq 1, U \subset Q(a, b)$ be a connected open set, and $f: U \rightarrow Q(A, B)$ be $a$ real-analytic $C R$ mapping such that $f(U)$ does not lie in a complex hyperplane then

$$
A \leq N(a, b, B)
$$

where $N=N(a, b, B)$ is a constant depending only on $a, b$, and $B$.

Proof. Let $\varphi=(f, g)$ denote a real-analytic CR mapping of $U \subset Q(a, b)$ to $Q(A, B)$, where $f: U \rightarrow \mathbb{C}^{A}$ and $g: U \rightarrow \mathbb{C}^{B}$. Plugging into the defining equation of the target hyperquadric we obtain that for $z \in U$

$$
\|f(z)\|^{2}-\|g(z)\|^{2}-1=0 .
$$

Real-analytic CR mappings extend to holomorphic mappings on a neighborhood of $U$. Therefore we assume that $f$ and $g$ are holomorphic in a neighborhood of $U$. We let $r(z, \bar{z})=$ $\|f(z)\|^{2}-\|g(z)\|^{2}-1$.

The condition that $\varphi(U)$ does not lie in a complex hyperplane is simply stating that components of the mapping $(f, g, 1)$ are linearly independent. In particular we see that $r$ has rank $A+B+1$, and signature pair $(A, B+1)$.

We apply Lemma 6.2 to $r$ to obtain

$$
A \leq K_{b, a+b}(B+1)
$$




\section{Spheres AND hyperquadrics in Hilbert SPACE}

Forstnerič [11] has shown that there exist strictly pseudoconvex real-analytic compact hypersurfaces that cannot be embedded into a sphere of any finite dimension. On the other hand, Lempert [16] has shown that a mapping exists if one takes the sphere in the infinite dimensional Hilbert space $\ell^{2}$. Let us make the following natural definitions to extend hyperquadrics to $\ell^{2}$ :

$$
\begin{aligned}
& Q(\infty, b):=\left\{z \in \ell^{2}:-\sum_{j=1}^{b}\left|z_{j}\right|^{2}+\sum_{j=b+1}^{\infty}\left|z_{j}\right|^{2}=1\right\} \\
& Q(\infty, \infty):=\left\{z \in \ell^{2}: \sum_{j=1}^{\infty}\left(\left|z_{2 j-1}\right|^{2}-\left|z_{2 j}\right|^{2}\right)=1\right\}
\end{aligned}
$$

We write $S^{\infty}=Q(\infty, 0)$ for the unit sphere in $\ell^{2}$.

By a real-analytic CR mapping from a CR manifold $M$ to $Q(\infty, b)$ we mean a holomorphic mapping to $\ell^{2}$ defined on a neighborhood of $M$ taking $M$ to $Q(\infty, b)$.

We use the ideas from the previous section to construct mappings. For example, consider the Hermitian form

$$
r(z, \bar{z})=\frac{e^{\left|z_{1}\right|^{2}+\left|z_{2}\right|^{2}}-1}{e-1}-1 .
$$

The function $r$ vanishes precisely on the unit sphere in $\mathbb{C}^{2}$. In fact $\frac{e^{\left|z_{1}\right|^{2}+\left|z_{2}\right|^{2}}-1}{e-1}$ is 1 on the unit sphere and is positive semi-definite. Hence $r$ has signature pair $(\infty, 1)$; that is, $r$ has infinitely positive eigenvalues and exactly one negative eigenvalue corresponding to the -1 . The form induces a real-analytic CR mapping

$$
f: S^{2} \rightarrow S^{\infty}
$$

We expand the series:

$$
\begin{aligned}
\frac{e^{\left|z_{1}\right|^{2}+\left|z_{2}\right|^{2}}-1}{e-1}= & \frac{\left(\left|z_{1}\right|^{2}+\left|z_{2}\right|^{2}\right)}{e-1}+\frac{\left(\left|z_{1}\right|^{2}+\left|z_{2}\right|^{2}\right)^{2}}{2(e-1)}+\frac{\left(\left|z_{1}\right|^{2}+\left|z_{2}\right|^{2}\right)^{3}}{6(e-1)}+\cdots \\
= & \left|\frac{z_{1}}{\sqrt{e-1}}\right|^{2}+\left|\frac{z_{2}}{\sqrt{e-1}}\right|^{2}+ \\
& \quad+\left|\frac{z_{1}^{2}}{\sqrt{2(e-1)}}\right|^{2}+\left|\frac{z_{1} z_{2}}{\sqrt{2(e-1)}}\right|^{2}+\left|\frac{z_{2}^{2}}{\sqrt{2(e-1)}}\right|^{2}+\cdots
\end{aligned}
$$

Therefore

$$
\begin{aligned}
f\left(z_{1}, z_{2}\right)=\left(\frac{z_{1}}{\sqrt{e-1}}, \frac{z_{2}}{\sqrt{e-1}},\right. & \frac{z_{1}^{2}}{\sqrt{2(e-1)}}, \frac{z_{1} z_{2}}{\sqrt{2(e-1)}}, \frac{z_{2}^{2}}{\sqrt{2(e-1)}}, \\
& \left.\frac{z_{1}^{3}}{\sqrt{6(e-1)}}, \frac{z_{1}^{2} z_{2}}{\sqrt{6(e-1)}}, \frac{z_{1} z_{2}^{2}}{\sqrt{6(e-1)}}, \frac{z_{2}^{3}}{\sqrt{6(e-1)}}, \ldots\right) .
\end{aligned}
$$

The image is not contained in a hyperplane; this fact can be seen directly by uniqueness of power series.

By taking

$$
r_{2}(z, \bar{z})=r(z, \bar{z})+|\varphi(z)|^{2} r(z, \bar{z})
$$


for an arbitrary holomorphic function $\varphi(z)$, we obtain a new form with signature pair $(\infty, 2)$, and therefore a CR mapping $f: S^{3} \rightarrow Q(\infty, 1)$, whose image is not contained in a hyperplane. By repeating the procedure we construct mappings

$$
f: S^{3} \rightarrow Q(\infty, b)
$$

for any finite $b$. Finally, by considering the form $r=\sin \left(\frac{\pi}{2}\left(\left|z_{1}\right|^{2}+\left|z_{2}\right|^{2}\right)\right)$ we obtain a mapping from $S^{3}$ to $Q(\infty, \infty)$.

As in the finite dimensional case, the situation changes when the source is a hyperquadric not equivalent to a sphere. If we apply Lemma 6.2, we notice the following immediate consequence.

Corollary 7.1. Let $a \geq 2, b \geq 1$. Let $U \subset Q(a, b)$ be a connected open set and $f: U \rightarrow$ $Q(\infty, B)$, where $B \in \mathbb{N}_{0} \cup\{\infty\}$, be a real-analytic $C R$ mapping such that $f(U)$ is not contained in any complex hyperplane of $\ell^{2}$. Then $B=\infty$.

Therefore, there is no mapping from $Q(a, b)$ for finite $a$ and $b$ into a $Q(\infty, B)$ for finite $B$. This rigidity result does not generalize to other hypersurfaces with indefinite nondegenerate Levi-form. In this case we can construct mappings to $Q(\infty, B)$ for finite $B$.

For example, define $r$ as

$$
r(z, \bar{z})=e^{\left|z_{1}+1\right|^{2}+\left|z_{2}\right|^{2}}-e-\left|z_{3}\right|^{2} .
$$

By expanding the series we see that the signature pair of $r$ is $(\infty, 2)$. Furthermore, we look at the series up to the quadratic terms

$$
r(z, \bar{z})=2 e \operatorname{Re} z_{1}+\left|z_{2}\right|^{2}-\left|z_{3}\right|^{2}+\text { higher order terms. }
$$

The Levi form of $M=\{r=0\}$ at the origin is indefinite; that is, it has one positive and one negative eigenvalue. As the signature pair is $(\infty, 2)$ we obtain a real-analytic CR mapping

$$
f: M \rightarrow Q(\infty, 1)
$$

whose image is not contained in a complex hyperplane. Similarly we can easily obtain a submanifold with $b$ negative eigenvalues and arbitrary number of positive eigenvalues in the Levi-form that admits a real-analytic CR mapping to $Q(\infty, b)$ whose image is not contained in a hyperplane.

The same construction can be used in the finite dimensional case. By truncating the series expansion of $r$, we obtain a polynomial $r^{\prime}$ and a manifold $M^{\prime}=\left\{r^{\prime}=0\right\}$ with an indefinite Levi-form at 0 , and such that $r^{\prime}$ has signature pair $(A, 2)$ for an arbitrarily high finite $A$. We obtain a mapping from $M^{\prime}$ to $Q(A, 1)$ whose image is not contained in a hyperplane.

\section{Construction of MAPpings}

Let us give some explicit examples in nonhomogeneous coordinates before we give a general method for constructing mappings.

Example 8.1. Let $(z, w) \in \mathbb{C}^{a} \times \mathbb{C}^{b}$. We tensor with the identity (which we write as $(z, w)$ ) on the $z_{a}$ variable. That is, we construct the mapping

$$
(z, w) \mapsto\left(z_{1}, \ldots, z_{a-1}, z_{a} \otimes(z, w), w\right) .
$$

We obtain a mapping taking $Q(a, b)$ to $Q(2 a-1,2 b)$. That is,

$$
(z, w) \mapsto\left(z_{1}, \ldots, z_{a-1}, z_{a} z_{1}, \ldots, z_{a}^{2}, z_{a} w_{1}, \ldots, z_{a} w_{b}, w_{1}, \ldots, w_{b}\right) .
$$


Therefore the super-rigidity result of Baouendi, Ebenfelt, and Huang [3] is sharp. In the same language as in the introduction we have that $A=2 a-1$ and $B=2 b$. On the other hand, by Theorem 1.3 we are not able to make $A$ arbitrarily large while keeping $B=2 b$.

Example 8.2. We could repeat the procedure of the first example any number of times. We take a mapping $\varphi: Q(a, b) \rightarrow Q(A, B)$ and construct

$$
\left(\varphi_{1}, \ldots, \varphi_{A} \otimes(z, w), \varphi_{A+1}, \ldots, \varphi_{A+B}\right) .
$$

We obtain

$$
\begin{aligned}
(z, w) \mapsto\left(\varphi_{1}(z, w), \ldots, \varphi_{A-1}(z, w), z_{1} \varphi_{A}(z, w), \ldots z_{a} \varphi_{A}(z, w)\right. & \\
& \left.w_{1} \varphi_{A}(z, w), \ldots w_{b} \varphi_{A}(z, w), \varphi_{A+1}(z, w), \ldots, \varphi_{A+B}(z, w)\right)
\end{aligned}
$$

taking $Q(a, b)$ to $Q(A+a-1, B+b)$.

For the constructions, it is easier to work in homogeneous coordinates. We define the homogeneous hyperquadric

$$
H Q(a, b):=\left\{z \in \mathbb{C}^{a+b}: \sum_{j=1}^{a}\left|z_{j}\right|^{2}-\sum_{j=a+1}^{a+b}\left|z_{j}\right|^{2}=0\right\} .
$$

The set $H Q(a, b)$ defines a real hypersurface in the projective space $\mathbb{C P}^{a+b-1}$. By setting $z_{a+b}=1$ we obtain $Q(a, b-1)$ in $\mathbb{C}^{a+b-1}$. The homogeneous setting is more symmetric, although one has to be careful when converting back to the nonhomogeneous situation.

Suppose that $r(z, \bar{z})$ is a bihomogeneous polynomial and vanishes on $H Q(a, b)$. If $r$ has signature pair $(A, B)$ (that is $A$ positive and $B$ negative eigenvalues), then we find homogeneous polynomials $f: \mathbb{C}^{a+b} \rightarrow \mathbb{C}^{A}$ and $g: \mathbb{C}^{a+b} \rightarrow \mathbb{C}^{B}$ with linearly independent components such that

$$
r(z, \bar{z})=\|f(z)\|^{2}-\|g(z)\|^{2}
$$

therefore the mapping $z \mapsto(f(z), g(z))$ takes $H Q(a, b)$ to $H Q(A, B)$. By dividing by $g_{B}$ and setting $z_{a+b}=1$ we obtain a rational CR mapping $F: Q(a, b-1) \rightarrow Q(A, B-1)$. If the components of $(f, g)$ are linearly independent then the image of $F$ does not lie in a complex hyperplane.

Therefore when constructing examples we will consider homogeneous polynomial mappings from $H Q(a, b)$ to $H Q(A, B)$ with linearly independent components.

\section{STABILITY}

We wish to show that as long as the number of positive and negative eigenvalues of the target hyperquadric grow in a comparable way, then nontrivial hyperquadric mappings always exist. We have the following stability result. We make the normalization $a \geq b$ for $H Q(a, b)$.

Theorem 9.1. Fix integers $a \geq b \geq 2$. There exists an $M\left(M=a^{2}+a b-2 a+1\right.$ suffices) such that when $A+B \geq M$, where $A, B \geq 2$ and

$$
\frac{B-b+1}{A} \geq \frac{b-1}{a} \quad \text { and } \quad \frac{A-b+1}{B} \geq \frac{b-1}{a},
$$

then there exists a homogeneous polynomial mapping $F: H Q(a, b) \rightarrow H Q(A, B)$ with linearly independent components. That is, there exists a rational $C R$ mapping $\tilde{F}: Q(a, b-1) \rightarrow$ $Q(A, B-1)$ whose image is not contained in a complex hyperplane. 
In [7] it is proved that when the source is a sphere (that is, $H Q(a, 1)$ ) then such a rational mapping always exists for large $A+B$ provided only that $A, B \geq 1$. In the hyperquadric case, this sort of stability result holds in a sector, where the size of the sector depends on $a$ and $b$. There may exist mappings outside of the sector given above; however, by Theorem 1.1 it is clear that any sector in which existence holds must make an acute angle.

The signature pairs of mappings that are constructed in the proof along with the inequalities are illustrated in Figure 1.



FiguRE 1. Diagram of mappings constructed in the proof of Theorem 9.1 for the source hyperquadric $H Q(4,2)$. The lattice represents the positive quadrant of the $(A, B)$ plane. A black dot represents that a mapping to $H Q(A, B)$ exists by applying the theorem. A black square represents that a mapping was also constructed in the proof. A circle represents a position in the lattice for which the theorem tells us nothing. The three lines represent the limits of the stability region from the theorem. The dashed squares represent one succession of the "squares" constructed in the proof.

Proof. Suppose $p(x)$ is a homogeneous polynomial in the variables $x_{1}, \ldots, x_{a+b}$ with $A$ positive and $B$ negative coefficients such that $p(x)=0$ when $x_{1}+\cdots+x_{a}-x_{a+1}-\cdots-x_{a+b}=0$. We call such polynomials admissible for $(A, B)$. We define

$$
s:=x_{1}+\cdots+x_{a}-x_{a+1}-\cdots-x_{a+b} .
$$

If $p$ is admissible for $(A, B)$ then we let $x_{k}=\left|z_{k}\right|^{2}$ in $p$. We obtain a Hermitian symmetric polynomial

$$
r(z, \bar{z})=p(x) .
$$

As distinct monomials are linearly independent, the rank of $r$ is equal to the number of distinct monomials of $p$, that is $A+B$ and the signature pair is $(A, B)$. As $r$ vanishes on $s=0$, we have that $r$ induces a CR mapping of $H Q(a, b)$ to $H Q(A, B)$ such that the image is not contained in a complex hyperplane.

Therefore we work with admissible polynomials $p$. We fix $a$ and $b$ and we work in the plane $\mathbb{N}_{0}^{2}$ and say $(A, B) \in E \subset \mathbb{N}_{0}^{2}$ whenever an admissible $p$ exists for $(A, B)$ (that is, a mapping to $H Q(A, B)$ exists $)$. 
We note that $(a, b) \in E$ and $(b, a) \in E$. If $p$ is admissible for $(A, B)$ of degree $m$ then $\tilde{p}=x_{1}^{k-m} p+x_{2}^{k-1} s$ vanishes when $s$ vanishes. If we pick $k$ large enough then the monomials of $x_{1}^{k-m} p$ and $x_{2}^{k-1} s$ are distinct and $\tilde{p}$ is admissible for $(A+a, B+b)$. By using $-s$ instead of $s$ we build an admissible polynomial for $(A+b, B+a)$. Therefore for any $n, k \in \mathbb{N}_{0}$

$$
(a, b)+n(a, b)+k(b, a) \in E \quad \text { and } \quad(b, a)+n(a, b)+k(b, a) \in E .
$$

We get a lattice of admissible points. We claim that for each point $(b, a)+n(a, b)+k(b, a)$ we also obtain that the "square"

$$
\{(b, a)+n(a, b)+k(b, a)-(j, m): 0 \leq j, m \leq n+k\}
$$

is a subset of $E$. Once we have the claim, it is a matter of noticing that the squares must overlap for large enough $n+k$. The inequalities from the theorem follow. See Figure 1 for illustration of the proof.

To prove the claim, we need to show that if $(A, B)$ is admissible, then the points

$$
\begin{array}{ll}
(A, B)+(a, b), & (A, B)+(a, b-1), \\
(A, B)+(a-1, b), & (A, B)+(a-1, b-1),
\end{array}
$$

and

$$
\begin{array}{ll}
(A, B)+(b, a), & (A, B)+(b, a-1), \\
(A, B)+(b-1, a), & (A, B)+(b-1, a-1) .
\end{array}
$$

are also admissible.

We have already seen that the first point belongs to $E$. Take $p$ that is admissible for $(A, B)$. By dividing through by $x_{a+b}$ we can suppose that $p$ contains a monomial $m$ that does not depend on $x_{a+b}$. Furthermore we assume that $m$ has a positive coefficient, since if no such monomial existed, setting $x_{a+b}=0$ would obtain a contradiction.

Write $p=m+q$ and construct

$$
\tilde{p}=m\left(s+x_{a+b}\right)+x_{a+b} q .
$$

Note that $s+x_{a+b}=x_{1}+\cdots-x_{a+b-1}$. The polynomial $\tilde{p}$ vanishes when $s$ vanishes. As the coefficient of $m$ is positive then $\tilde{p}$ is admissible for $(A, B)+(a-1, b-1)$.

Similarly we construct

$$
\hat{p}=\frac{m\left(s+x_{a+b}\right)}{2}+\frac{x_{a+b} m}{2}+x_{a+b} q .
$$

The polynomial $\hat{p}$ vanishes when $s$ vanishes. As the coefficient of $m$ is positive then $\tilde{p}$ is admissible for $(A, B)+(a, b-1)$.

All the other points in the claim follow by variations on the above construction.

\section{REFERENCES}

[1] N. I. Akhiezer and I. M. Glazman, Theory of linear operators in Hilbert space, Dover Publications Inc., New York, 1993. MR1255973

[2] M. Salah Baouendi, Peter Ebenfelt, and Linda Preiss Rothschild, Real submanifolds in complex space and their mappings, Princeton Mathematical Series, vol. 47, Princeton University Press, Princeton, NJ, 1999. MR1668103

[3] M. S. Baouendi, Peter Ebenfelt, and Xiaojun Huang, Holomorphic mappings between hyperquadrics with small signature difference, Amer. J. Math. 133 (2011), no. 6, 1633-1661, DOI 10.1353/ajm.2011.0044.

[4] M. S. Baouendi and Xiaojun Huang, Super-rigidity for holomorphic mappings between hyperquadrics with positive signature, J. Differential Geom. 69 (2005), no. 2, 379-398. MR2169869 
[5] John P. D'Angelo, Several complex variables and the geometry of real hypersurfaces, Studies in Advanced Mathematics, CRC Press, Boca Raton, FL, 1993. MR1224231

[6] John P. D'Angelo and Jiř́i Lebl, Complexity results for CR mappings between spheres, Internat. J. Math. 20 (2009), no. 2, 149-166. MR2493357

[7] _ Hermitian symmetric polynomials and CR complexity, J. Geom. Anal. 21 (2011), no. 3, 599-619. MR2810845

[8] John P. D'Angelo, Hermitian analogues of Hilbert's 17-th problem, Adv. Math. 226 (2011), no. 5, 46074637. MR2770459

[9] John P. D'Angelo and Dror Varolin, Positivity conditions for Hermitian symmetric functions, Asian J. Math. 8 (2004), no. 2, 215-231. MR2129535

[10] Franc Forstnerič, Extending proper holomorphic mappings of positive codimension, Invent. Math. 95 (1989), no. 1, 31-61. MR969413

[11] _ Embedding strictly pseudoconvex domains into balls, Trans. Amer. Math. Soc. 295 (1986), no. 1, 347-368, DOI 10.2307/2000160. MR831203

[12] Mark Green, Generic initial ideals, Six lectures on commutative algebra (Bellaterra, 1996), Progr. Math., vol. 166, Birkhäuser, Basel, 1998, pp. 119-186. MR1648665

[13] - Restrictions of linear series to hyperplanes, and some results of Macaulay and Gotzmann, Algebraic curves and projective geometry, 1989, pp. 76-86. MR1023391

[14] Xiaojun Huang, Shanyu Ji, and Dekang Xu, A new gap phenomenon for proper holomorphic mappings from $B^{n}$ into $B^{N}$, Math. Res. Lett. 13 (2006), no. 4, 515-529. MR2250487

[15] Jiří Lebl and Daniel Lichtblau, Uniqueness of certain polynomials constant on a line, Linear algebra Appl. 433 (2010), 824-837. MR2654111

[16] László Lempert, Imbedding Cauchy-Riemann manifolds into a sphere, Internat. J. Math. 1 (1990), no. 1, 91-108, DOI 10.1142/S0129167X90000071. MR1044662

[17] Hans Lewy, On the local character of the solutions of an atypical linear differential equation in three variables and a related theorem for regular functions of two complex variables, Ann. of Math. (2) 64 (1956), 514-522. MR0081952

Department of Mathematics, University of Michigan, Ann Arbor, Mi 48109, USA

E-mail address: grundmer@umich.edu

Department of Mathematics, University of Wisconsin, Madison, WI 53706, USA

E-mail address: lebl@math.okstate.edu

Department of Mathematics, Purdue University, West Lafayette, IN 47907, USA

E-mail address: lvivas@math.purdue.edu 